\title{
Modelling the Impact of Pericyte Migration and Coverage of Vessels on the Efficacy of Vascular Disrupting Agents
}

\author{
S.R. McDougall ${ }^{1}$, M.A.J. Chaplain ${ }^{2 *}$, A. Stéphanou ${ }^{3}$ and A.R.A. Anderson ${ }^{2}$ \\ ${ }^{1}$ Heriot-Watt University, Edinburgh, EH14 4AS, Scotland \\ ${ }^{2}$ Division of Mathematics, University of Dundee, Dundee, DD1 4HN, Scotland \\ ${ }^{3}$ Faculté de Médecine de Grenoble, 38706 La Tronche Cedex, France.
}

\begin{abstract}
Over the past decade or so, there have been a large number of modelling approaches aimed at elucidating the most important mechanisms affecting the formation of new capillaries from parent blood vessels - a process known as angiogenesis. Most studies have focussed upon the way in which capillary sprouts are initiated and migrate in response to diffusible chemical stimuli supplied by hypoxic stromal cells and leukocytes in the contexts of solid tumour growth and wound healing. However, relatively few studies have examined the important role played by blood perfusion during angiogenesis and fewer still have explored the ways in which a dynamically evolving vascular bed architecture can affect the distribution of flow within it. From the perspective of solid tumour growth and, perhaps more importantly, its treatment (e.g. chemotherapy), it would clearly be of some benefit to understand this coupling between vascular structure and perfusion more fully. This paper focuses on the implications of such a coupling upon chemotherapeutic, anti-angiogenic, and anti-vascular treatments.

In an extension to previous work by the authors, the issue of pericyte recruitment during vessel maturation is considered in order to study the effects of different anti-vascular and anti-angiogenic therapies from a more rigorous modelling standpoint. Pericytes are a prime target for new vascular disrupting agents (VDAs) currently in clinical trials. However, different compounds attack different components of the vascular network and the implications of targeting only certain elements of the capillary bed are not immediately clear. In light of these uncertainties, the effects of anti-angiogenic and anti-vascular drugs are re-examined by using an extended model that includes an interdependency between vessel remodelling potential and local pericyte density. Two- and three-dimensional simulation results are presented and suggest that it may be possible to identify a VDA-specific "plasticity window" (a time period corresponding to low pericyte density), within
\end{abstract}

\footnotetext{
*Corresponding author. E-mail: chaplain@maths.dundee.ac.uk
} 
which a given VDA would be most effective.

Key words: tumour-induced angiogenesis, blood vessel networks, pericytes, chemotherapeutic, anti-angiogenic, anti-vascular treatment protocols

AMS subject classification: 35Q35, 35Q92, 65M06, 92C17, 92C35, 92C50

\section{Introduction}

Uncontrolled or excessive blood-vessel formation is an essential accompaniment to solid tumour growth, beginning with the rearrangement and migration of endothelial cells from a pre-existing vasculature and culminating in the formation of an extensive network, or bed, of new capillaries [30] (Madri and Pratt, 1986). Although the precise molecular cascades associated with a given instance of angiogenesis may differ from case to case, a common sequence of events associated with tumour-induced angiogenesis has been broadly identified and well-documented.

The process begins when the oxygen demands of cancerous cells within a solid tumour are unable to be adequately met via diffusion from nearby capillaries. These cells consequently become hypoxic and this is assumed to trigger cellular release of tumour angiogenic factors (TAFs) [15] (Folkman and Klagsbrun, 1987), which start to diffuse into the surrounding tissue and approach the endothelial cells of nearby blood vessels. These endothelial cells subsequently respond to the TAF concentration gradient by releasing a number of matrix degrading enzymes (including matrix metalloproteinases), which degrade the surrounding tissue leading to the formation of new capillary sprouts. These then migrate towards the tumour [43] [4] [44] (Schoefl, 1963; Ausprunk and Folkman, 1977; Sholley et al. 1984) and the resulting vascular connection subsequently provides all the nutrients and oxygen required for continued tumour growth. Initially, the sprouts arising from the parent vessel grow in an essentially parallel manner. However, once the finger-like capillary sprouts have reached a certain distance from the parent vessel, they are seen to incline toward each other [34] (Paweletz and Knierim, 1989), leading to numerous tip-to-tip and tip-to-sprout fusions known as anastomoses. Such anastomoses result in the fusing of the finger-like sprouts into a network of poorly perfused loops or arcades. Following this process of anastomosis, the first signs of circulation can be recognised and from the primary loops, new buds and sprouts emerge repeating the angiogenic sequence of events and providing for the further extension of the new capillary bed.

Most modelling studies dealing with the process of angiogenesis have tended to concentrate upon the way in which the new capillary bed is initiated and migrates in response to various chemical stimuli and mechanical forces affiliated with the tumour and host tissue. However, relatively few studies have examined the important role played by blood perfusion during angiogenesis and fewer still have explored the ways in which a dynamically evolving bed architecture can affect the distribution of flow within it. This is clearly an important feature of angiogenesis, as capillary size and bed architecture are key determinants of not only oxygen delivery to the tumour during growth, but also chemotherapy delivery during treatment. It is reasonable to assume that an aberrant tumour vasculature will hinder uniform delivery of therapeutic compounds to the tumour tissue.

Although there have been a number of theoretical models developed to try to better understand 
vascular architecture in general [42] [18] [25] (Secomb, 1995; Gödde and Kurz, 2001; Krenz and Dawson, 2002) and to examine clinical implications in a broader sense [6] [14] [22] [39] (Baish et al, 1996; El-Kareh and Secomb, 1997; Jackson, 1999; Quarteroni, 2000), there have only been a small number of theoretical studies examining blood flow in tumour-induced (micro) capillary networks [28] [1] [45] [46] [29] (McDougall et al., 2002; Alarcon et al., 2003; Stéphanou et al., 2005a, b; McDougall et al., 2006). Any therapeutic applications described in these studies have tended to focus upon the impact of bed topology on the delivery of a cytotoxic drug to a tumour and have largely neglected the important possibility of disrupting the vascular network itself. The fact that new treatments are being tailored to specifically target vascular endothelium suggests that it would be of some benefit to understand the coupling between vascular structure and perfusion more fully in this new context.

Genetically, vascular cells are more stable than tumour cells. The latter tend to mutate during chemotherapy, leading to increased levels of resistance to infused cytotoxic compounds [33] (Munn, 2003), and it is the relative genetic stability of endothelial cells that makes them ideal candidates for treatment. The vast majority of solid tumours depend upon a local blood supply for continued expansion, and so one naïve standpoint to the issue of cancer treatment may be to simply search for the most effective "capillary killer". There are a number of difficulties with this approach, however. Firstly, tumour-induced vasculature is often composed of several different cell types - not only endothelial cells, but also fibroblasts, pericytes, and even tumour cells themselves. In addition, the relative proportions of each cell type in the cellular mosaic tends to vary from tissue to tissue. Secondly, different vascular disrupting agents (VDAs) attack vascular networks in different ways. For example, combrestatins act as tubulin depolymerisation agents and primarily attack only proliferating endothelial cells [48] (Tozer et al, 2005), whilst other compounds act as angiogenesis inhibitors by blocking the signalling pathways associated with various growth factors [9] [21] (Brekken and Thorpe, 2001, Izumi et al, 2002). Additional VDAs currently under investigation include compounds that affect the down-regulation of integrin activity [26] (Kumar et al, 2000) and the recruitment of circulating endothelial precursor cells [40] (Rafil et al, 2002).

The fact that different VDAs target different elements of the vascular network suggests that it is not too surprising that the overall effectiveness of any one drug would be case-specific. Moreover, the wider implications of targeting only certain elements of the capillary bed are not immediately clear. However, it appears that there is one characteristic of a developing capillary network that may be correlated with VDA efficacy — namely, pericyte coverage. First described by Rouget in 1873 [41], the pericyte is a perivascular cell that is recruited by migrating capillaries, helping to stabilise the network structure by wrapping finger-like extensions around the developing vessels. Although its ontogeny remains somewhat controversial, it is clear that the pericyte plays a vital role during angiogenesis and there is growing evidence that disrupting pericyte-endothelium interactions may inhibit tumour growth [17] [5] (Gee et al, 2003; Bagley et al, 2006). Moreover, it appears that VDAs that target only endothelial cells may leave behind an intact skeleton of pericytes that could act as a template for revascularisation at a later time [31] (Mancuso et al, 2006).

In light of these issues, it is clear that the time is ripe for the development of a modelling framework that incorporates not only important feedback mechanisms associated with blood flow 
through evolving vascular networks, but also the recruitment of pericytes during vessel maturation. This paper accordingly describes the development of such a framework and presents a range of twoand three-dimensional numerical simulations that are used to identify the main criteria required of a tumour-induced vascular network for optimised delivery of cytotoxic, anti-angiogenic, and/or anti-vascular agents. The ultimate aim of the approach is to identify a VDA-specific "plasticity window" (a time period corresponding to low pericyte density), within which a given VDA would be most effective.

The next section briefly outlines the capillary growth model used throughout this work, the precise details of which are summarised in Appendix A. The model is an extension of that proposed earlier by Anderson and Chaplain (1998) [2] and incorporates the important feature of matrix degrading enzyme secretion by migrating endothelial cells. This discussion is then followed by a short précis describing the introduction of blood perfusion and vessel dilation/constriction into the model. A fuller description, including details of the algorithms used to capture the effects of blood rheology, vessel remodelling, and shear-induced vessel branching, is presented in Appendix B. Capillary beds corresponding to different stages in the development of the fully dynamic adaptive tumour-induced angiogenesis (DATIA) model are presented. The results show the evolution of the model and demonstrate the impact of dynamic remodelling and shear-induced vessel branching upon global network architecture.

Having summarised the angiogenesis model itself, attention is next focussed upon the utilisation of the model for the simulation of various chemotherapy treatment protocols. Two- and three-dimensional results relating to the delivery of chemotherapeutic agents to the tumour surface are presented in Section 3: firstly via a static, uniform bed of rigid capillaries, then via a dynamically adaptive bed. Results from even the simple static model show the importance of tumour geometry and anastomosis density in determining treatment efficacy, whilst results from the full DATIA model demonstrate the impact of vessel heterogeneity within a given network. These simulations highlight the need for incorporating vessel adaptations into any angiogenesis model involving transport issues, such as chemotherapeutic intervention, and also show that three-dimensional modelling is required if quantitative predictions are to be made.

Section 4 considers the effects of anti-angiogenic and anti-vascular therapies - i.e. therapies that are specifically aimed at disrupting the vascular bed itself. These are initially modelled by means of simple "capillary pruning" algorithms that are used to approximate the key mechanisms associated with different treatments. The pruning algorithms are seen to modify the network connectivity in a number of different ways and the impact of each upon subsequent chemotherapy delivery is discussed. Finally, a model for pericyte recruitment to migrating capillaries is described and the spatial distribution of pericyte density is investigated in both two and three dimensions under a number of different model assumptions, including cases where pericyte coverage (i.e. vessel maturity) determines the degree of vessel remodelling. Results suggest that it may indeed be possible to identify a "plasticity window" that would maximise the effectiveness of a given VDA.

The paper concludes with a discussion section summarising all the main results and offering directions for future model development and study. 


\section{Dynamic Adaptive Tumour-Induced Angiogenesis (DATIA)}

This section begins by briefly describing the salient features of the extended angiogenesis model (including the effects of matrix degrading enzymes) used to migrate capillary sprouts from a nearby parent vessel. This is followed by a short summary describing the way in which blood perfusion and vessel dilation/constriction mechanisms have been incorporated into the model. These extensions result in a more realistic model of the angiogenesis process that is both adaptive and dynamic, with the resulting vascular network evolving both temporally and spatially in response to a number of migratory and transport-related cues. Results are presented that demonstrate the importance of these processes in determining global network architecture.

\subsection{Capillary Migration in the Absence of Flow}

The dynamic capillary migration model presented in this paper explicitly takes into account the important function of matrix degrading enzymes (such as matrix metalloproteinases, MMPs; urokinase plasminogen activators, uPAs) during angiogenesis in the absence of flow [27] [11] (Levine et al., 2001; Lolas, 2003). Mediation in vessel growth via extracellular matrix proteolysis by specific enzymes produced by endothelial cells is also included. A number of recent publications have demonstrated the importance of enzymes from the MMP family and their involvement in the regulation of the various stages of the angiogenic process [13] [49] [19] [47] (Davis et al., 2000; Yan et al., 2000; Hidalgo and Eckhardt, 2001; Sternlicht and Werb, 2001). These MMPs are involved in the migration of endothelial cells within the extracellular matrix, endothelial cell proliferation, and the remodelling of the basement membrane of newly formed vessels. Their importance is such that these proteinases and their regulation form new targets for cancer treatment. As our ultimate goal is to propose a global modelling framework within which to further investigate new treatments, it is important to incorporate the MMP effect into the modelling.

All of the vasculatures presented in this paper were generated using a hybrid discrete-continuum model inspired by the tumour-induced angiogenesis model proposed by Anderson and Chaplain (1998) [2]. The model assumes that endothelial cells at the tips of the new capillary sprouts (vessels) migrate through (i) random motility, (ii) chemotaxis in response to tumour angiogenic factors (TAF) released by the tumour and (iii) haptotaxis in response to fibronectin (FN) gradients in the extracellular matrix. A (nondimensional) equation describing endothelial cell $(n)$ conservation is used to produce movement weightings for discrete tip cells as they leave the parent vessel (see Appendix A and Figure A1). The equation is given by:

$$
\frac{\partial n}{\partial t}=\overbrace{D \nabla^{2} n}^{\text {random }}-\overbrace{\nabla \cdot(\chi(c) n \nabla c)}^{\text {chemotaxis }}-\overbrace{\rho \nabla \cdot(n \nabla f)}^{\text {haptotaxis }},
$$

In addition to an equation for endothelial cell density, the model also requires equations governing the evolution of angiogenic factor $(c)$, matrix degrading enzyme $(m)$, and the matrix-bound protein associated with the haptotactic response (fibronectin in this case, denoted by $f$ ). For the TAF, it is assumed that a (quasi) steady state distribution already exists in the matrix, the TAF having initially been secreted by the tumour cells. As the endothelial cells migrate through the tissue, there is some 
binding of the TAF to the cells and this is modelled with a simple uptake term that is switched on locally in the presence of a migrating tip cell. Fibronectin exists in the matrix in bound form and therefore there is no diffusion term for fibronectin. Endothelial cells are known to produce fibronectin as they migrate and also to degrade the matrix as they progress. Consequently, simple production and loss terms in the fibronectin equation are switched on wherever an endothelial tip cell exists to reflect these facts. Tumour angiogenesis factors and fibronectin are known to bind to specific membrane receptors on endothelial cells and subsequently trigger molecular cascades inside the ECs, activating cell migratory machinery. One consequence of this activation process is the production by the cells of a matrix degrading enzyme (MDE), which enhances the attachment of the cells to fibronectin contained in the extracellular matrix. The endothelial cells are consequently able to exert the traction forces required to propel themselves during migration. This mechanism is included in the modelling by allowing individual tip cells to produce MDE locally, which then diffuses and degrades within the host tissue. With these modelling assumptions the full (nondimensional) system of equations can be defined as:

$$
\begin{aligned}
& \frac{\partial c}{\partial t}=-\eta n_{k} c, \\
& \frac{\partial f}{\partial t}=\beta n_{k}-\gamma m f, \\
& \frac{\partial m}{\partial t}=\alpha n_{k}+\varepsilon \nabla^{2} m-\nu m .
\end{aligned}
$$

where $c$ represents the TAF concentration, $f$ the FN concentration, $m$ the MDE density and $n_{k}$ a Boolean value ( 1 or 0 ) that indicates the presence or absence of an endothelial cell at a given position. Details of other parameters, together with a brief explanation of the hybrid modelling approach and algorithms for sprout branching and anastomosis are given in Appendix A.

\subsection{Capillary Migration Incorporating Flow}

Having extended the migration model to account for the key biochemical interactions characterising the angiogenesis process, attention next turns to the incorporation of perfusion-related mechanisms. Previous approaches examining flow through tumour-induced networks by [28] McDougall et al. (2002) and [45] Stéphanou et al. (2005a) had made the rather limiting assumptions of constant capillary radius and invariant blood viscosity, whereas, in reality, biological structures tend to exhibit some degree of compliance and blood is non-Newtonian . This earlier formulation must therefore be extended to account for variable blood viscosity and evolving capillary vessels that may either dilate or constrict both spatially and temporally. The details of how this has been achieved, including a discussion related to the different timescales associated with endothelial cell migration and capillary flow processes, are available in [29] McDougall et al (2006) and will only be briefly summarised here. 


\section{Blood Rheology}

Because of its biphasic nature, blood does not behave as a continuum and the viscosity measured while flowing at different rates in microvessels is not constant. Moreover, direct measurement of blood viscosity in living microvessels is very difficult to achieve with any degree of accuracy. Pries et al. (1996) [35] have proposed an alternative approach, which involves "history matching" the flow distribution in a numerical network (generated by a mathematical model) with similar experimental systems. The relationship which was found to offer the best fit with the experimental data at the microvascular scale, is given by:

$$
\mu_{\text {rel }}\left(R, H_{D}\right)=\left[1+\left(\mu_{0.45}-1\right) f\left(H_{D}\right)\left(\frac{2 R}{2 R-1.1}\right)^{2}\right]\left(\frac{2 R}{2 R-1.1}\right)^{2}
$$

where $\mu_{0.45}$ is the viscosity corresponding to the normal average value of the discharge haematocrit $\left(H_{D}=0.45\right), \mathrm{R}$ the vessel radius and $f\left(H_{D}\right)$ a function of the haematocrit. The various terms appearing in (2.3) are defined as follows:

$$
\begin{aligned}
& \mu_{0.45}=6 e^{-0.17 R}+3.2-2.44 e^{-0.06(2 R)^{0.645}}, \\
& f\left(H_{D}\right)=\frac{\left(1-H_{D}\right)^{C}-1}{(1-0.45)^{C}-1} \quad, \\
& C=\left(0.8+e^{-0.15 R}\right)\left(-1+\frac{1}{1+10^{-11}(2 R)^{12}}\right)+\left(\frac{1}{1+10^{-11}(2 R)^{12}}\right) .
\end{aligned}
$$

The relative viscosity defined by equations (2.3) and (2.4) has been used in an extended form of Poiseuille's law at the scale of a single capillary. Of course, this is only one particular formulation of relative viscosity and any well-founded local flow/pressure-drop relationship could easily be incorporated into the model if alternatives emerge from future experimental studies. The methodology used to model perfusion within a developing network is described more fully in [28] McDougall et al (2002) and summarised in Appendix B.

\section{Vessel Adaptation}

Blood rheological properties and microvascular network remodelling are interrelated issues, as blood flow creates stresses on the vascular wall (shear stress, pressure, tensile stress) which lead to adaptation of the vascular diameters via either vasodilatation or constriction. In turn, blood rheology (viscosity, haematocrit, etc.) is affected by the new network architecture - consequently, adaptive angiogenesis should be expected to be a highly dynamic process. In this paper, vessel adaptation follows the treatment of Pries et al. [35, 36, 37] $(1995,1998,2001$ a) and considers a number of stimuli affecting vessel diameter that account for the influence of the wall shear stress $\left(S_{w s s}\right)$, the intravascular pressure $\left(S_{p}\right)$, and a metabolic mechanism depending on the blood haematocrit $\left(S_{m}\right)$. These stimuli form a basic set of requirements in order to obtain stable network structures with realistic distributions of vessels diameters and flow velocities. The equation used 
for radial variation as a function of these stimuli is as follows:

$$
\Delta R=[\underbrace{\log \left(\tau_{w}+\tau_{r e f}\right)}_{S_{w s s}}-\underbrace{k_{p} \log \tau_{e}(P)}_{S_{p}}+\underbrace{k_{m} \log \left(\frac{Q_{r e f}}{Q H_{D}}+1\right)}_{S_{m}}-k_{s}] R \Delta t
$$

The various terms used in (2.5) are described more fully in [29] McDougall et al (2006) and a brief summary is supplied in Appendix B, along with details of a modified branching algorithm that accounts for vessel branching in areas of heightened wall shear stress.

\subsection{Bed Comparisons}

A comparison of the final vasculatures resulting from the different stages of the model development is shown in Figure 1 (2D) and Figure 2 (3D). Figure 1(a) shows a 2D network resulting from the simple migration model, without any flow-related remodelling, whilst Figure 1(b) demonstrates the effect of dynamic remodelling after growth. Although the overall architectures from the static and a posteriori remodelled approaches are similar, the dilated backbone apparent in the remodelled network will clearly play a dominant role in determining drug delivery to the tumour surface (as will be shown in the next section). Hence, it seems reasonable to infer that the effect of capillary remodelling should be incorporated into angiogenesis models at this scale, if transport issues are to be addressed.

Of course, restricting the remodelling of a capillary bed until after migration is complete is rather artificial, as blood perfusion begins soon after the first capillary arcade has formed. In reality, bed remodelling and capillary dilation/constriction occur as immediate consequences of primary anastomosis and the network resulting from this approach is shown in Figure 1(c). The effect of increased vessel branching in areas of high wall shear stress modifies the bed topology, leading to the formation of dilated arcades closer to the parent vessel accompanied by an overall increase in capillary density. The implications for therapeutic delivery of coupling capillary growth and radial adaptation will be discussed in the following section.

In addition to radial adaptation, the dimensionality of the capillary network itself will also be shown to have a large impact upon treatment delivery. The architecture comparison shown in Figure 2 suggests that drug bypassing could be even more of an issue in three dimensions, with the dilated backbone (Figure 2(c)) branching into many alternative directions and forming a dense brush border as the tumour periphery is approached. It would be reasonable to anticipate that delivery using 2D models would overestimate delivery considerably and that 3D models should be used for quantitative comparisons with experiment.

\section{Chemotherapy Delivery to the Tumour Periphery}

As a first attempt towards modelling the flow of chemotherapy drugs through tumour-induced vascular networks, an algorithm was developed to track concentration profiles of a generic tracer 


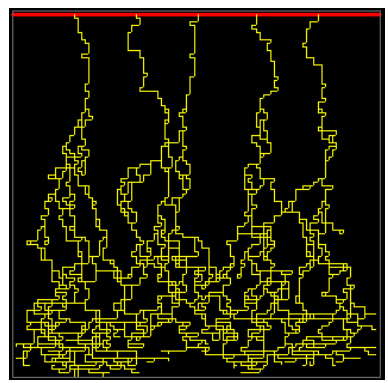

(a)

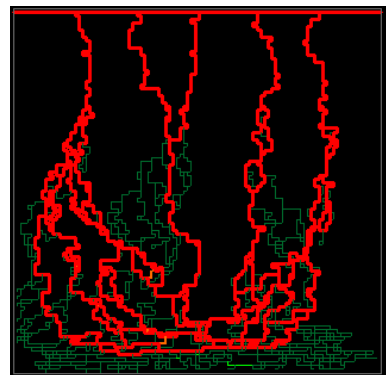

(b)

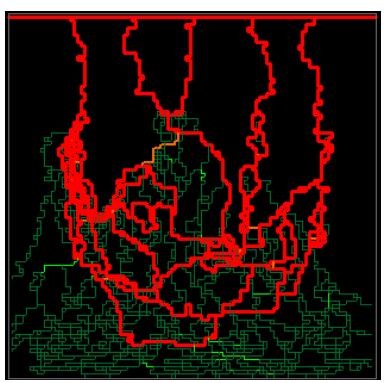

(c)

Figure 1: 2D capillary networks formed as endothelial sprouts migrate from a parent vessel at the upper boundary of the domain through the extracellular matrix in response to gradients in TAF (chemotaxis) and FN (haptotaxis). Growth complete after approximately 16 days.(a) Simple migration model without flow-induced remodelling of the capillaries; (b) a posteriori remodelling after growth; (c) full DATIA model, including shear-stress-induced branching. Radii vary from $12 \mu \mathrm{m}$ (red) to $6 \mu \mathrm{m}$ (yellow) to $2 \mu \mathrm{m}$ (green).

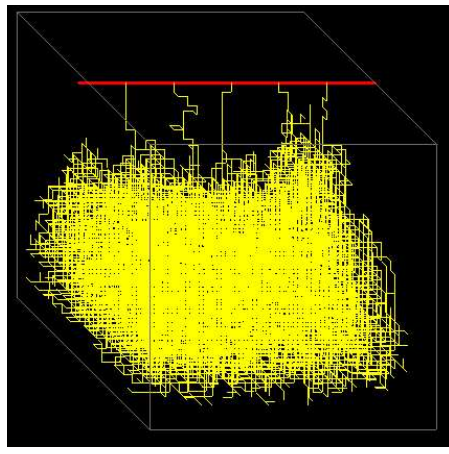

(a)

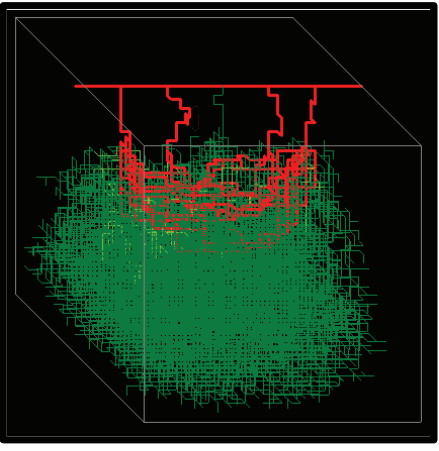

(b)

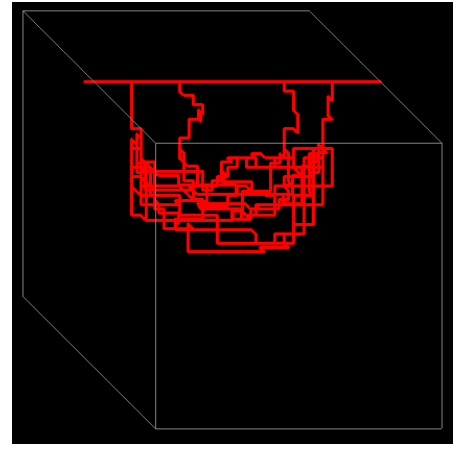

(c)

Figure 2: 3D capillary networks formed as endothelial sprouts migrate from a parent vessel at the upper face of the domain through the extracellular matrix. (a) Simple migration model without flow-induced remodelling of the capillaries; (b) full DATIA model, including shear-stress-induced branching; (c) the dilated backbone isolated from (b). Radii vary from $12 \mu \mathrm{m}$ (red) to $6 \mu \mathrm{m}$ (yellow) to $2 \mu \mathrm{m}$ (green). 
(drug) injected into the upstream end of the parent vessel. Initially, a constant viscosity was assumed for the circulating fluid, although this assumption was later relaxed when blood rheological properties were taken into account. In both instances, however, tracer concentrations were updated as follows. Firstly, nodal pressures and elemental flows were calculated within the tortuous network structure as described in Appendix B. At each timestep, the following procedure was adopted:

(i) the total amount of drug flowing into each node was calculated;

(ii) perfect mixing was assumed at each node and new drug concentrations were calculated for all outflow vessels based upon the updated nodal values.

It should be noted that the time-step required when dealing with calculations involving convective transport within a network must be less than or equal to the minimum time required for the least efficient capillary element in the network to empty; i.e. $\Delta \mathrm{t}=\mathrm{MIN}\left(\mathrm{V}_{\text {cap }} / \mathrm{Q}_{\text {cap }}\right)$, where $\mathrm{V}_{\text {cap }}$ and $\mathrm{Q}_{\text {cap }}$ correspond to the volume and flow of a capillary element. This procedure ensures that mass is conserved during a simulation. In the simulations described below, the lower edge of the domain is taken to represent the tumour periphery and it is assumed that any drug reaching this lower boundary is effectively delivered to the tumour mass itself. Of course, this is not strictly correct, as the pressure distribution within the tumour (as well as the resident vasculature network) will determine the actual degree of drug uptake. Hence, even the low uptake values shown below should be considered as maximum achievable cytotoxic doses.

The investigation of chemotherapeutic delivery to the tumour periphery begins with flow simulations on a static capillary bed comprising identical, rigid cylindrical vessels.

\subsection{Chemotherapy Delivery Via Static Capillary Beds}

The chemotherapy simulations presented in this section were undertaken using only a static, nonadaptive capillary bed and Newtonian fluid. The first set of results corresponds to continuous drug infusion into a 2D vascular network, which was generated by a linear source of TAF lying along the lower boundary of the domain. A chemotherapy drug of concentration $\mathrm{C}_{\max }$ was fed into the left-hand side of the parent vessel at $\mathrm{t}=0$ and sequential drug concentration profiles for the base case simulation are shown in Figure 3. It is clear that, instead of supplying drug to the tumour, some branches actually remove drug from the capillary network (Figure 3(c)), thereby reducing its efficacy. Indeed, for this set of input data, 16,800 seconds (4-5h) of continuous infusion is required for a drug concentration of $0.1 \% \mathrm{C}_{\max }$ to reach the tumour.

The effect of blood viscosity upon drug delivery and uptake is evident from the results shown in Figure 4(a). The drug reaches the tumour sooner when the blood viscosity is decreased, as may be inferred from the inverse relationship between elemental flow and fluid viscosity, coupled with the linear nature of the pressure equations. Next, by decreasing the mean capillary radius, the effect of narrowing the capillaries on drug deliverywas examined. Figure 4(b) shows the results of drug delivery into capillaries with a mean radius of $2 \mu \mathrm{m}, 3 \mu \mathrm{m}$ and $4 \mu \mathrm{m}$ (used in the base case). As the radius decreases the flow rate and consequently drug uptake by the tumour decreases. Hence for narrower capillaries infusion would have to continue for many hours/days before significant tumour uptake could occur. 
The second set of results using the simple static network corresponds to a 30-second bolus injection of drug into a network formed in response to a more focussed semicircular TAF source. The rather striking drug evolution snapshots are shown in Figure 5. The large number of interconnections between capillaries - even quite far from the tumour - means that there is very little flow in the lower part of the network, and the bolus of drug essentially bypasses the tumour.

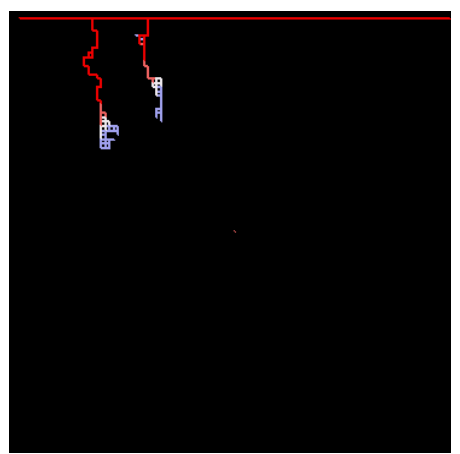

(a)

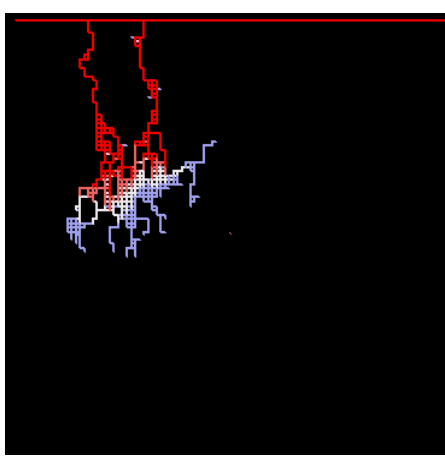

(b)

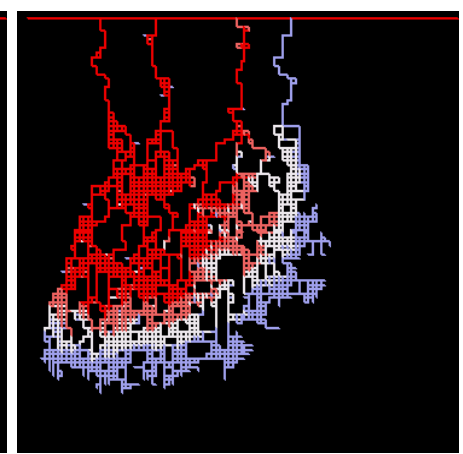

(c)

Figure 3: Concentration contours for continuous infusion into a vasculature formed from a linear TAF source. $100 \times 100$ domain size. (a) $t=240 s$, (b) $t=1200$ s, (c) $t=7200$ s. Red corresponds to high concentration and blue to low concentration.

Of course, in vivo tumour-induced vasculatures grow in three dimensions, and so it is important to ascertain whether the same bypassing issues apply. The snapshots shown in Figure 6 suggest that they do and a direct comparison between deliveries in a 2D and 3D system confirm this (Figure 7). Delivery is two orders of magnitude lower in the 3D case. This will be revisited in the context of a fully adaptive network in the next section.

\subsection{Chemotherapy Delivery Via Dynamically Adaptive Capillary Beds}

Results presented in this section will show the impact of dynamic remodelling and shear-induced vessel branching upon global network architecture and chemotherapeutic treatment delivery these highlight a number of new therapeutic targets for tumour management. The initial focus will be on two-dimensional capillary beds, as results are far easier to visualise and interpret. However, quantitative analysis requires the third dimension and so some drug delivery comparisons from 3D simulations will be presented at the end of the section.

An example of the changes seen in bed topology due to dynamic radial adaptation was presented earlier in Figure 1. The most important aspect of this example was that it demonstrated how shear-induced branching could lead to earlier formation of dilated anastomoses close to the parent vessel. However, this example is only one of many - by varying a number of physical and biochemical parameters in the model, a wide range of network heterogeneity is predicted (Figure 8). Consequently, by quantifying the efficiency of these different networks in carrying bloodborne material to the tumour, it is hoped that some insights can be offered into the precise fate of chemotherapeutic agents in the vasculature during treatment and, moreover, that this could lead 


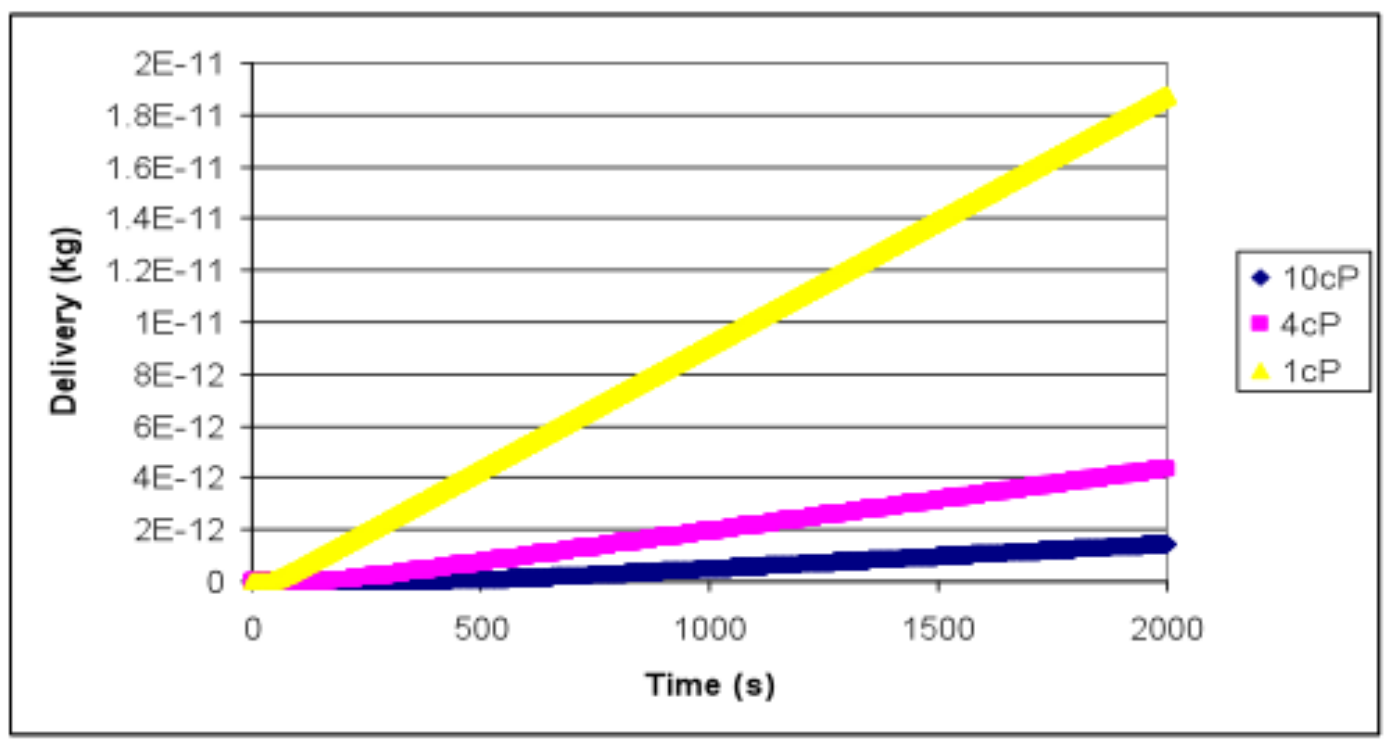

(a)

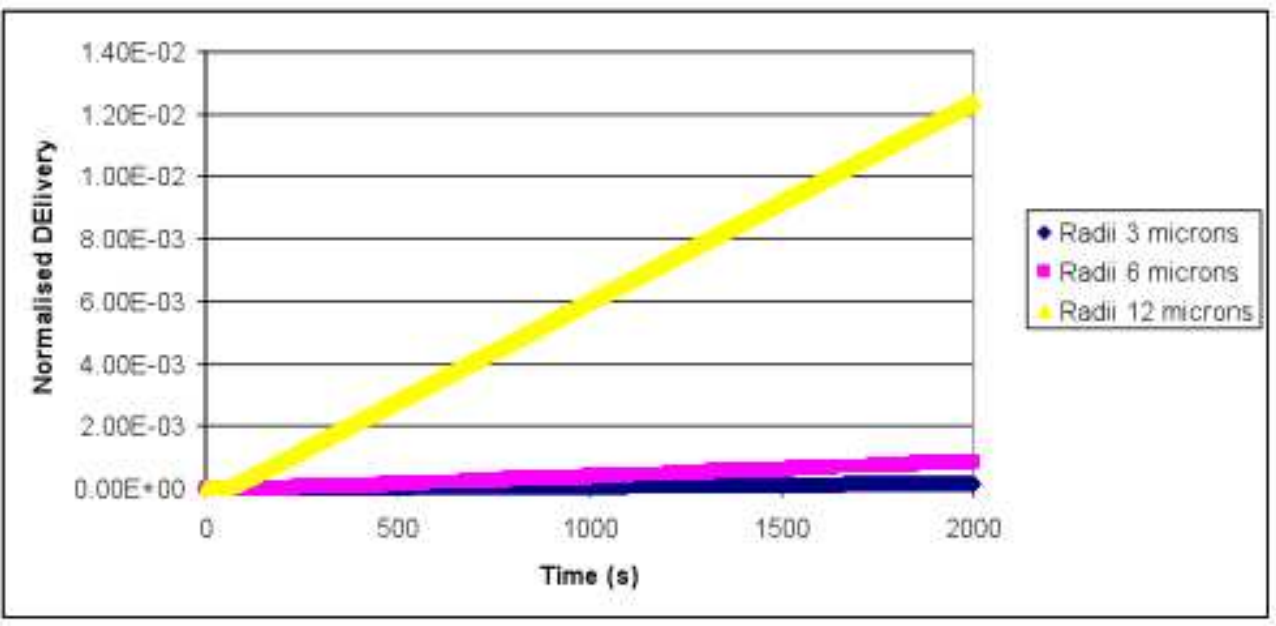

(b)

Figure 4: Effect of (a) viscosity, and (b) mean capillary radius, upon drug delivery - continuous infusion into a 30x30 vasculature formed from a linear TAF source. Delivery has been normalised in (a) to the total mass of drug injected into the parent vessel over 2000s. 


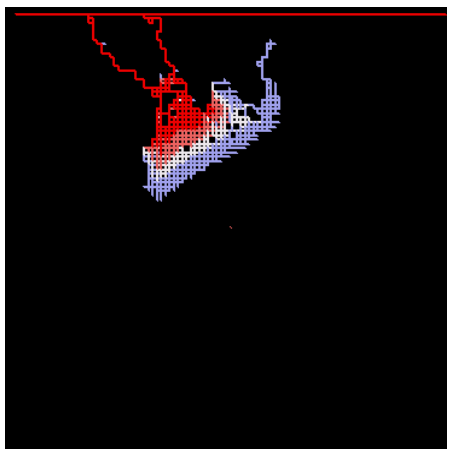

(a)

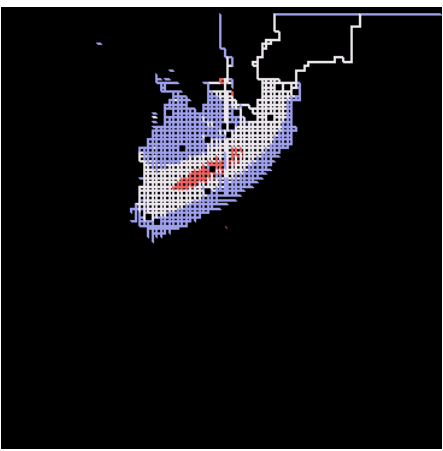

(b)

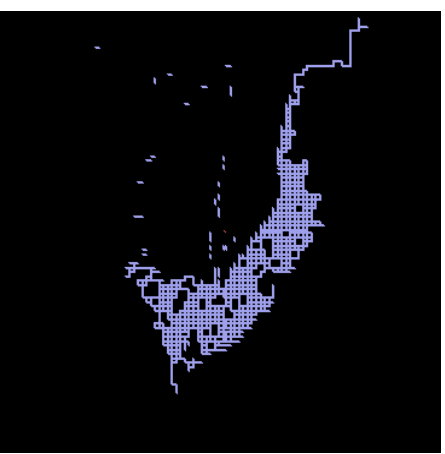

(c)

Figure 5: Concentration contours for bolus injection into a vasculature formed from a semicircular TAF source at the lower domain boundary. 100x100 domain size. (a) $t=1200 s$, (b) $t=3600 s$, (c) $\mathrm{t}=62$ 400s. Red corresponds to high concentration and blue to low concentration.

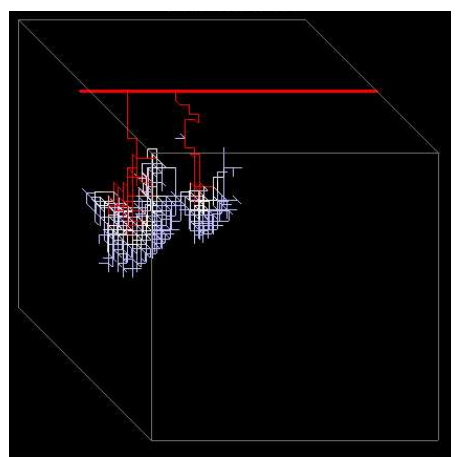

(a)

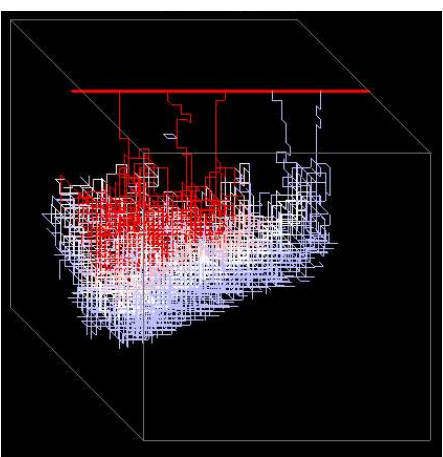

(b)

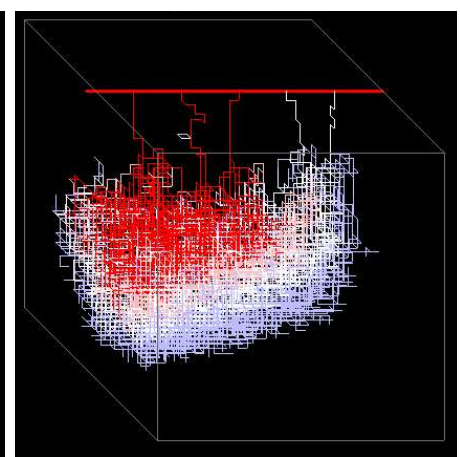

(c)

Figure 6: Concentration contours for continuous infusion into a $3 \mathrm{D}$ vasculature formed from a linear TAF source. $30 \times 30 \times 30$ domain size. (a) $t=50 \mathrm{~s}$, (b) $t=500 \mathrm{~s}$, (c) $t=1000$ s. Red corresponds to high concentration and blue to low concentration. 


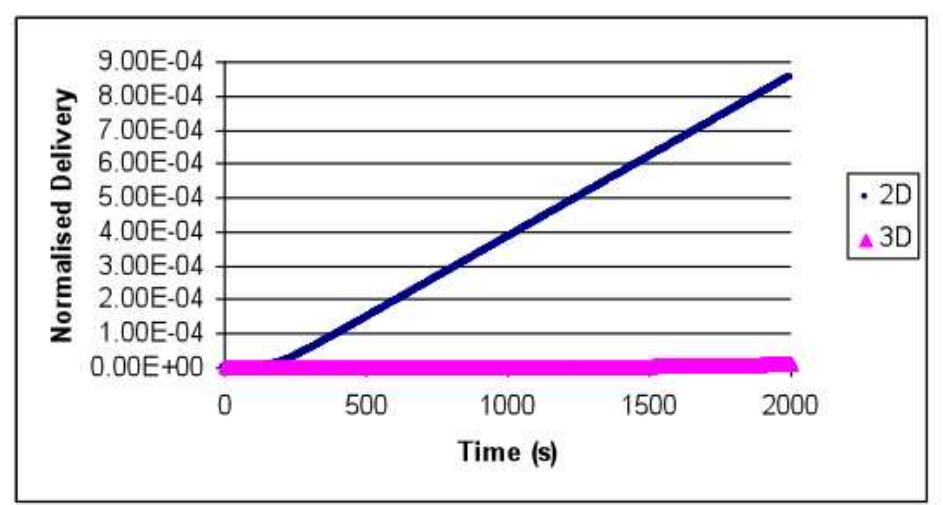

Figure 7: Normalised drug delivery comparison between a 30x30 static 2D vascular bed and a 30x30x30 static 3D vascular bed. Delivery has been normalised to the total mass of drug injected into the parent vessel over 2000s.

to the identification of a number of new therapeutic targets and strategies for tumour management (for example, drug-induced normalization of tumour blood vessels, to be addressed later in Section 4)

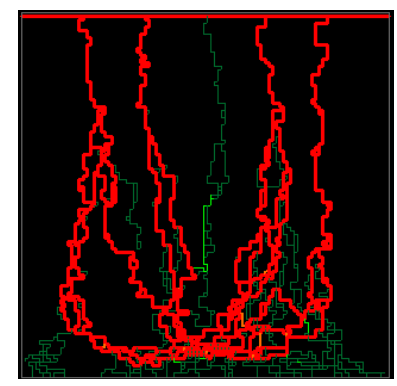

(a)

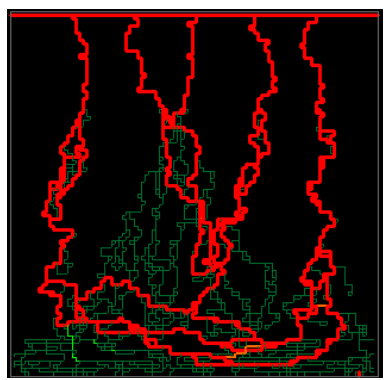

(c)

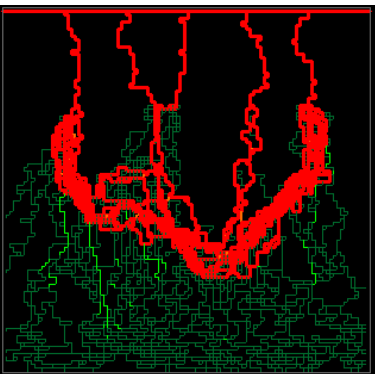

(b)

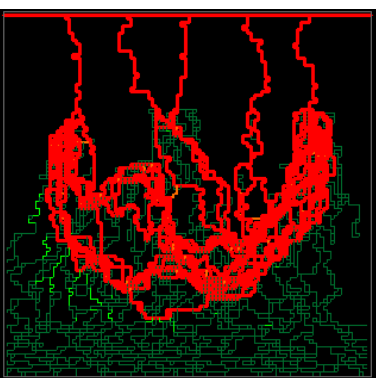

(d)

Figure 8: Plots showing the different steady-state capillary network structures which have evolved after 48 days due to changes in model parameters. (a) reduced haptotactic coefficient $\rho=0.16$; (b) reduced input haematocrit $\mathrm{H}_{D}=0.225$; (c) reduced inlet blood pressure $\left(\mathrm{P}_{i n}=2960 \mathrm{~Pa}\right.$, $\left.\mathrm{P}_{\text {out }}=2060 \mathrm{~Pa}\right)$; (d) reduced outlet blood pressure $\left(\mathrm{P}_{\text {in }}=3260 \mathrm{~Pa}, \mathrm{P}_{\text {out }}=1760 \mathrm{~Pa}\right)$. Compare with the base case in Figure 1(c). 
In order to assess transport efficacy within a given adapted vessel network, a drug at concentration $\mathrm{C}_{\max }$ was continuously infused into the inlet of the parent vessel for 500 seconds. The base-case simulation for transport utilised the vasculature shown in Figure 1(c). Figure 9 shows the tracer-drug evolution through the capillary network at a number of different times (in seconds). It is immediately clear that the bulk (in fact, almost all) of the injected tracer-drug flows through the highly conductive dilated backbone, largely by-passing the tumour and recirculating to the parent vessel. In excess of 250s of continuous infusion is required before any tracer-drug reaches the tumour surface, and only then in very small concentrations. Figure 10 shows plots of the total drug mass in the system (parent vessel and capillary network) and delivery to the tumour surface as functions of time. It should be noted that all masses have been normalised to the total mass injected into the parent vessel over the course of the simulation. Only around $1.5 \%$ of the infused tracer-drug even enters the capillary network and, although the total mass in the network reaches a plateau after approximately 50s (transport being essentially governed by steady-state flow through the dilated backbone), it takes another 200-250s before uptake commences. This is because capillaries forming part of the brush border close to the tumour surface are narrow and poorly perfused - consequently, only a very small fraction of the injected treatment actually reaches the target. As an aside, it should be noted that, although convective transport through the vessels of the network would be a rather poor delivery mechanism for large molecules (i.e. cytotoxic treatments), the dilated network is sufficiently well-developed within a few hundred microns of the tumour surface that diffusion of nutrients (oxygen, glucose) would be relatively efficient over the timescale of tumour growth.

Figure 11(a) shows the uptake using an identical network architecture but with all capillary radii set permanently to $6 \mu \mathrm{m}$ (the default value given at vessel birth in the model) - uptake values are approximately three orders of magnitude larger that those obtained from the remodelled vasculature. These results clearly demonstrate the impact of network heterogeneity upon treatment efficacy and highlight the need for incorporating vessel adaptations (dilation/constriction) into any angiogenesis model involving transport issues, such as chemotherapeutic intervention. In the absence of vessel size variation, delivery is greatly overestimated.

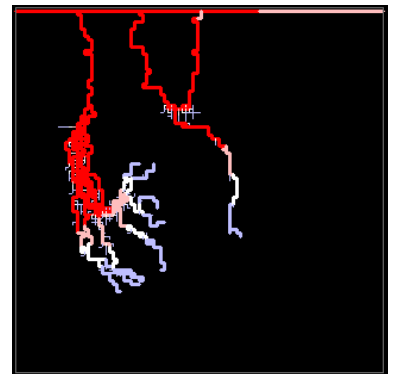

$\mathrm{t}=10$

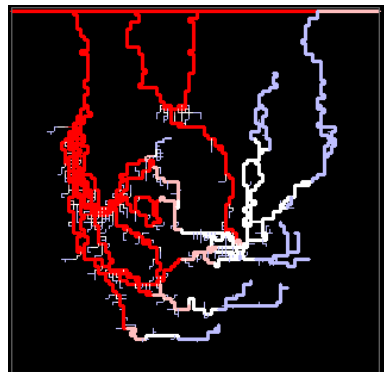

$\mathrm{t}=20$

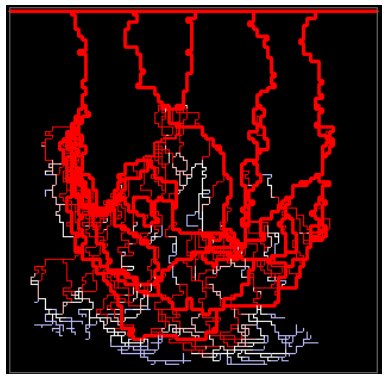

$\mathrm{t}=300$

Figure 9: Plots of the tracer-drug concentration distribution in the network shown in Figure 1(c) at different times. Red corresponds to high concentration and blue to low concentration.

A possible therapeutic target identified from the simulation is the manipulation of the haptotac- 


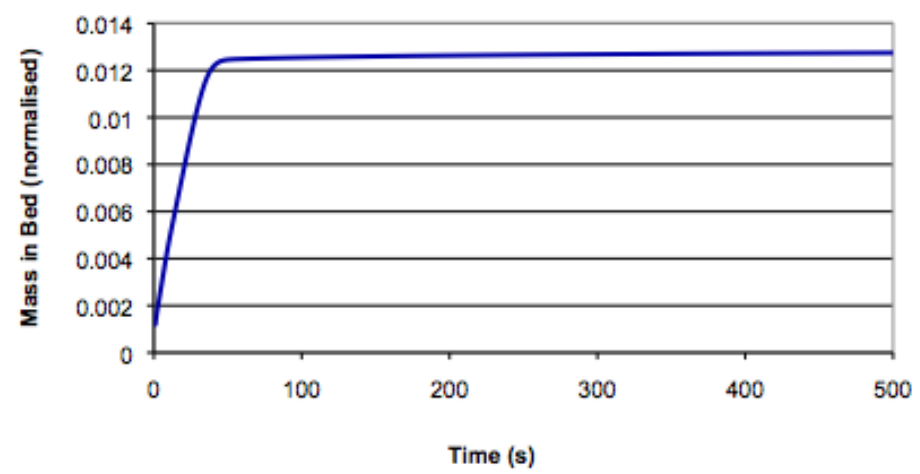

(a)

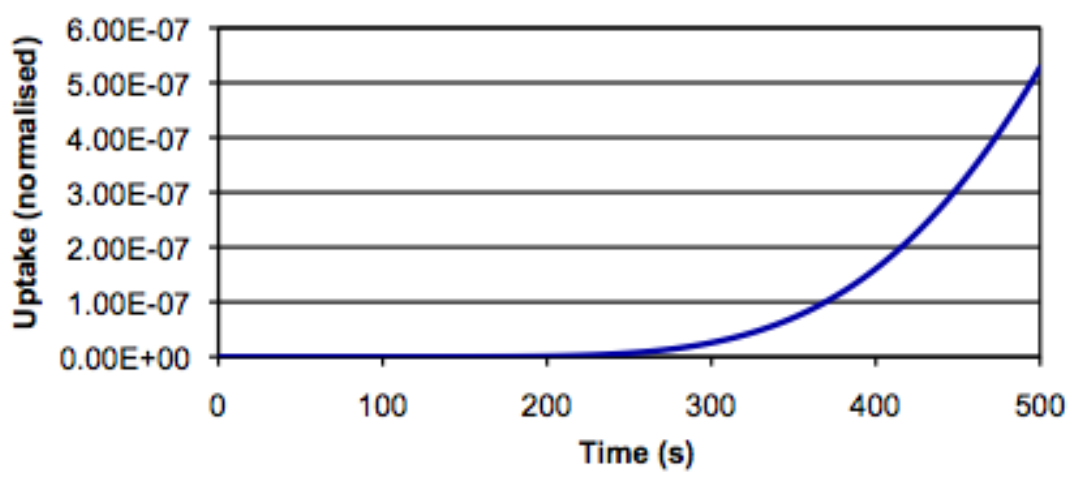

(b)

Figure 10: Plots of (a) normalised total drug mass in parent vessel and network over time and (b) normalised delivery reaching the tumour.

tic response of the migrating endothelial cells during angiogenesis, characterised by reduced lateral migration of the vessels and reduced shear-induced branching (Figure 8(a)). The tracer-drug evolution through this vessel network suggests that tumours supplied by this type of vasculature would be well-supplied with nutrients and could be expected to grow rapidly. Paradoxically, however, such tumours would also be highly susceptible to infused treatments, with far more cytotoxic agent reaching the tumour than observed in previous cases. This conjecture is supported by the uptake results from the infusion simulation shown in Figure 11(b). Whilst the total mass of tracer-drug entering the supplying vasculature is almost identical to that observed in the base case simulation ( $\rho=0.28$, not shown), the drug uptake by the tumour is fifty times greater when lateral migration and vessel branching are reduced.

The network shown in Figure 8(b) suggests that a depressed haematocrit can be expected to lead to the formation of highly dilated arcades close to the parent vessel and Figure 11(c) shows the therapeutic implications of this phenomenon - more drug enters the capillary network than entered in the base-case simulation but drug delivery to the tumour is reduced by more than three orders of magnitude. In the context of nutrient supply to the tumour, it is proposed here that 


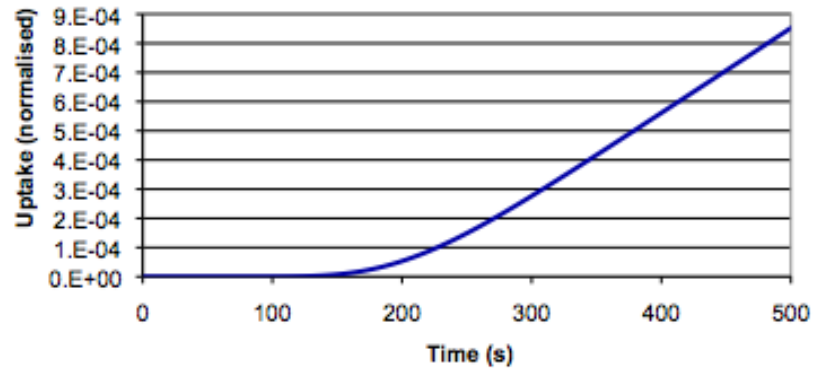

(a)

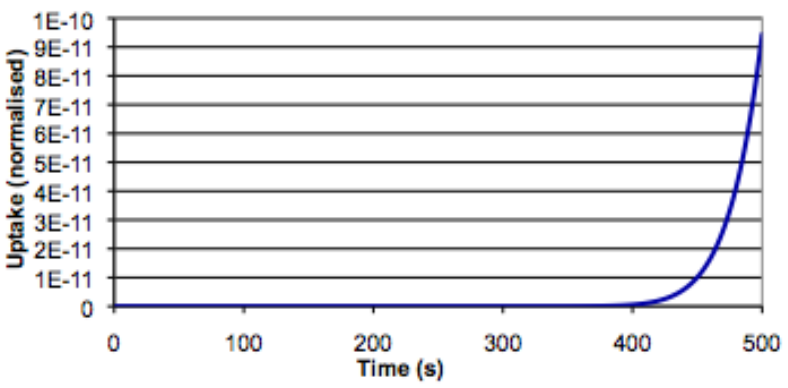

(c)

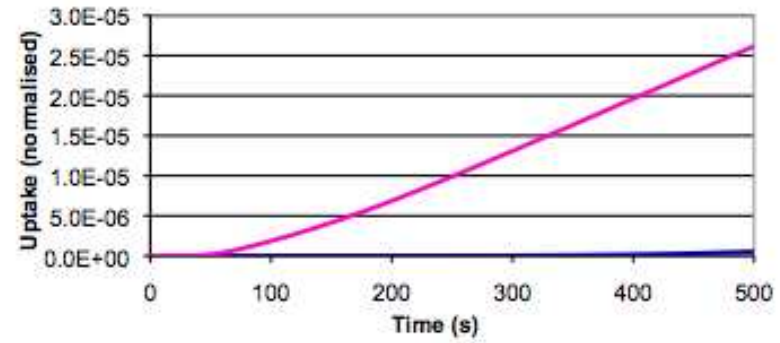

(b)

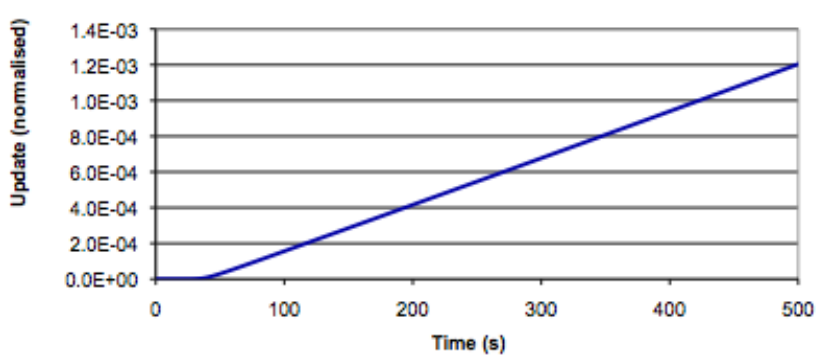

(d)

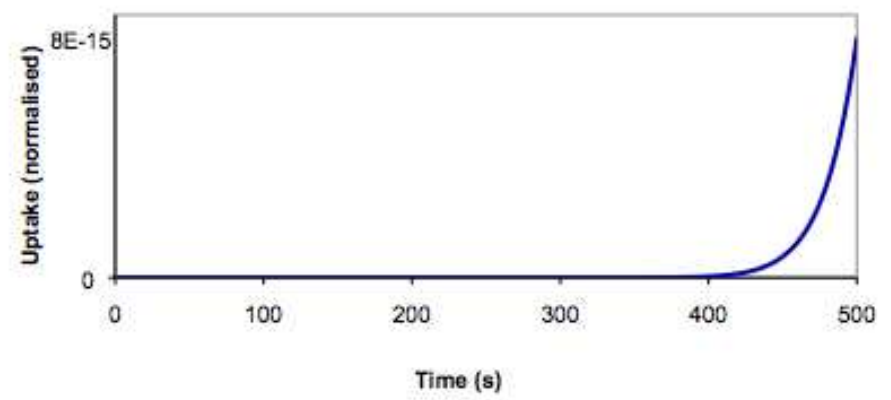

(e)

Figure 11: Plot showing the amount of drug reaching the tumour over time under various model assumptions. (a) a network where all vessels have a fixed radius of $6 \mu \mathrm{m}$. Note the difference in scale compared to that of Figure 10b. (b) the effect of varying haptotactic sensitivity (dashed line corresponds to $\rho=0.16$, solid line corresponds to $\rho=0.16$ ), (c) the effect of reduced input haematocrit $\mathrm{H}_{D}=0.225$, (d) lower pressure at inlet of parent vessel, (e) lower pressure at outlet of parent vessel.

decreasing local haematocrit could be a possible mechanism for generating vasculatures that are detrimental to tumour growth.

Figure 11(d) shows the impact upon drug delivery of lowering the pressure at the inlet of the parent vessel by $300 \mathrm{~Pa}(2.25 \mathrm{mmHg})$ prior to angiogenesis, whilst keeping the outlet pressure unchanged. Delivery is dramatically increased - by more than three orders of magnitude 
- and tumours characterised by similar vascular architectures are consequently highly likely to be vulnerable to chemotherapeutic infusions. Finally, Figure 11(e) shows the uptake by the tumour supplied by the network in Figure 8(d), where the pressure gradient across the parent vessel was increased by $300 \mathrm{~Pa}(2.25 \mathrm{mmHg})$ prior to angiogenesis. Uptake is extremely poor, which is not too surprising given the presence of highly-dilated loops close to the parent vessel. Hence, intravenous/intra-arterial treatments would be expected to prove ineffective in this case.

Having examined the role played by vessel adaptation on drug delivery qualitatively in twodimensions, attention next turns towards quantitative simulations in three dimensions. Snapshots of the drug evolution through the 3D adaptive bed are shown in Figure 12 and once again demonstrate the dominant role played by the dilated backbone in determining drug bypassing. As expected, bypassing begins earlier in the adaptive network, with the backbone effectively at maximum concentration after approximately 100 seconds. Delivery comparisons between 3D adaptive and non-adaptive networks and between 2D and 3D adaptive networks are presented in Figure 13. Increased dimensionality and vessel adaptation each reduce delivery by over an order of magnitude. The importance of the third dimension and the inclusion of adaptive architecture in providing quantitative predictions for comparison with in vivo results is clear.

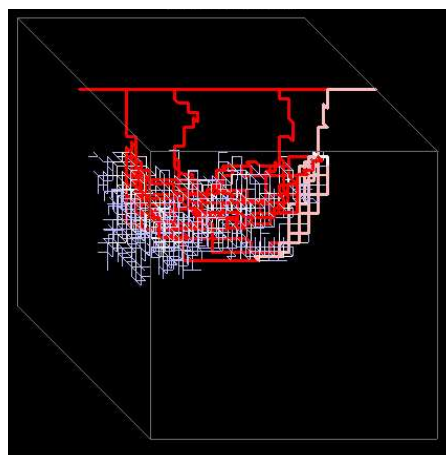

(a)

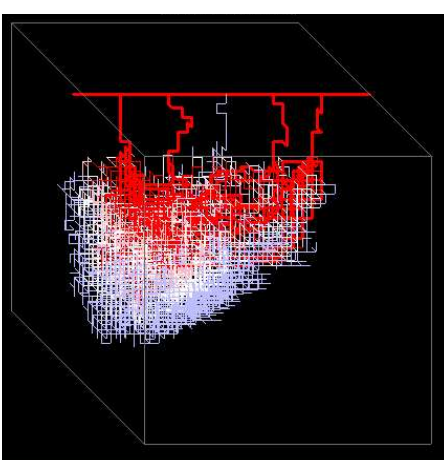

(b)

Figure 12: Concentration contours for continuous infusion into an adaptive 3D vasculature formed from a linear TAF source. 30x30x30 domain size. (a) $t=50 \mathrm{~s}$, (b) $\mathrm{t}=500 \mathrm{~s}$. Red corresponds to high concentration and blue to low concentration.

\section{Anti-Angiogenic and Anti-Vascular Treatment Simulations}

The previous section described the delivery of chemotherapeutic agents from parent vessel to tumour boundary and did not discuss the possibility of certain drugs modifying the capillary architecture itself. In this section, several models are presented to examine precisely this issue - i.e. the effects of anti-angiogenic and anti-vascular therapies that are specifically aimed at disrupting the vascular bed. These therapies are initially modelled by means of simple "capillary pruning" algorithms that are used to approximate the key mechanisms thought to pertain to different treatments. 


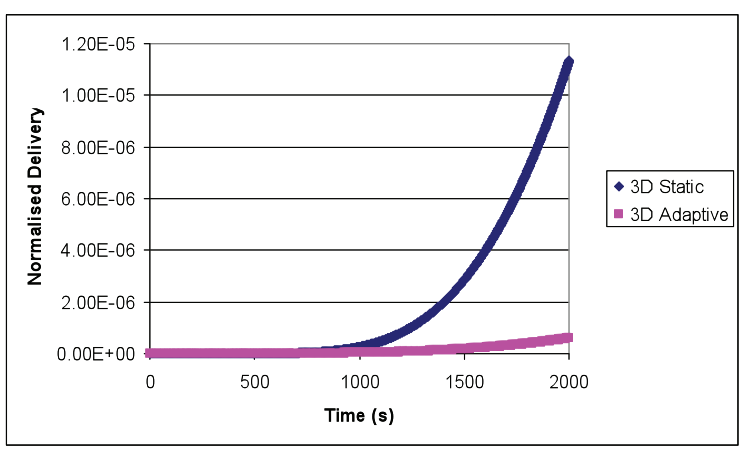

(a)

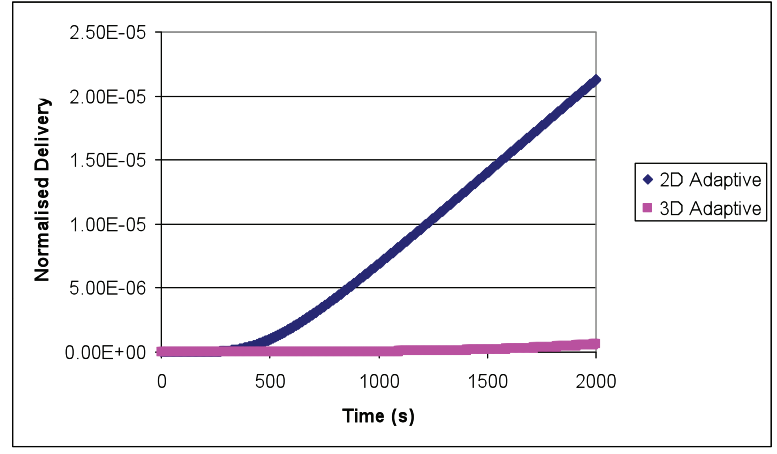

(b)

Figure 13: Normalised drug delivery comparisons. (a) 30x30x30 static 3D bed vs 30x30x30 adap-

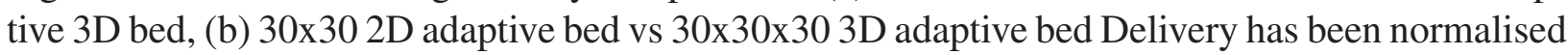
to the total mass of drug injected into the parent vessel over 2000 s.

Attention is restricted to 2D vasculatures for ease of presentation and interpretation, although similar qualitative conclusions can be immediately inferred for three dimensions.

The capillary pruning approach could be considered as being somewhat naïve, however, in that the underlying biological principles of how a certain therapy operates is subsumed into a very basic vessel removal algorithm. Hence, in order to study the effects of different anti-vascular and anti-angiogenic therapies from a more rigorous modelling standpoint, a new model is described that explicitly incorporates pericyte recruitment to migrating capillaries. The spatial distribution of pericyte density is investigated in both 2D and 3D under a number of different model assumptions and the implications for anti-angiogenic and anti-vascular treatments are discussed.

\subsection{Capillary Pruning}

In this section, the effect of various modifications to the network connectivity upon the efficiency of drug delivery to the tumour is investigated. The motivation for this comes from the possibility of targeting particular areas of a growing vasculature with anti-angiogenic and anti-vascular drugs. This section focuses upon a posteriori capillary pruning (i.e. capillary removal from a static preexisting capillary bed) of a vasculature similar to that shown in Figure 1(a). Modifications are achieved by removing a fraction of vessels in the lower part of the vasculature.

Three pruning algorithms have been considered: (i) vessels removed in a totally random fashion; (ii) vessels removed if their flow lies below a given threshold; (iii) vessels removed if their flow exceeds a given threshold. These three approaches seek to broadly cover the spectrum of possible targeting strategies for anti-angiogenic and anti-vascular treatments - the first relates to a broad-based indiscriminate anti-vascular drug, the second relates to an anti-angiogenic drug that preferentially targets immature, poorly perfused capillaries, and the third relates to the possible targeting of capillaries characterized by high wall stresses. In all simulations, a bolus injection at concentration $C_{\max }$ and duration $t=375 \mathrm{~s}$ was considered. 


\section{Random vessel removal}

Figure 14 presents the variation in drug uptake at time $t=2500 \mathrm{~s}$ as the fraction $(f)$ of vessels randomly removed from the initial vasculature is increased. Note, that $t=2500 \mathrm{scorresponds}$ to the time at which drug uptake via the unpruned vasculature (i.e. $f=0.0$ ) ceased. It can be seen in Figure 14 that the total amount of drug uptake increases to a maximum for $f=0.06$. A sharp decrease is then observed, corresponding to poorly connected architectures that are unable deliver drug effectively.

Visualization of the flow distribution in the optimized vasculature shows an increase in flow in the distal part of the capillary bed (close to the tumour) and this redistribution increases the efficacy of drug delivery to the tumour by approximately $130 \%$ (Figure 15). These simulations clearly show that drug delivery to a tumour becomes optimized when (i) highly interconnected regions of the vasculature are removed; and (ii) a high capillary density can be preserved close to the tumour surface. If an anti-angiogenic treatment could achieve this architecture, then the efficacy of subsequent chemotherapy treatments would be much improved. However, we should also point out that if an anti-angiogenic agent were to be used in isolation (i.e. without chemotherapeutic followup), then the effect may be to simply optimize nutrient supply and this could actually encourage tumour growth.

\section{Flow-dependent vessel removal: low flow rate threshold}

As shown above, the process of random removal of vessels can lead to drastic changes in drug delivery to a tumour and these variations can be either positive or negative. In this section, motivated by the possibility of targeting drugs at poorly perfused areas of the vasculature, some of the vessels are selectively removed if their flow falls below a given threshold value.

The next simulation focussed on a case where vessels having flow less than $1 \%$ of the maximum capillary flow $\left(Q_{\max }\right)$ were removed - the fraction removed actually represents $40 \%$ of the total capillary bed. It is found that the flow distribution remains essentially unchanged when compared with the unmodified vasculature and a comparison of drug uptake in the two systems shows that maximum uptakes are also similar (Figure 16). It should be noted, however, that the modified network initially delivers the drug more quickly: the "treated" vasculature has been optimized and drug delivery is accelerated by approximately $30 \%$.

\section{Flow-dependent vessel removal: high flow rate threshold}

The final approach to capillary pruning relates to the possible targeting of "bottleneck" capillaries characterized by high wall stresses. In this scenario, capillaries with flow exceeding $95 \%$ of the maximum network value were removed - this corresponded to a removed fraction $f=0.05$. Figure 17 shows the resulting delivery profile. It can be seen that the network has essentially shut down in this case, with no drug delivery to the tumour. Hence, if an anti-vascular treatment could be developed to target these fast-flowing "bottleneck" capillaries, it would be a highly efficient way of slowing tumour growth.

\subsection{Pericyte Recruitment and "Plasticity Window" Identification}

Having discussed a number of broad approximations to modelling the effects of anti-vascular and anti-angiogenic drugs, a more rigorous basis for vascular disruption is now sought. It is clear 


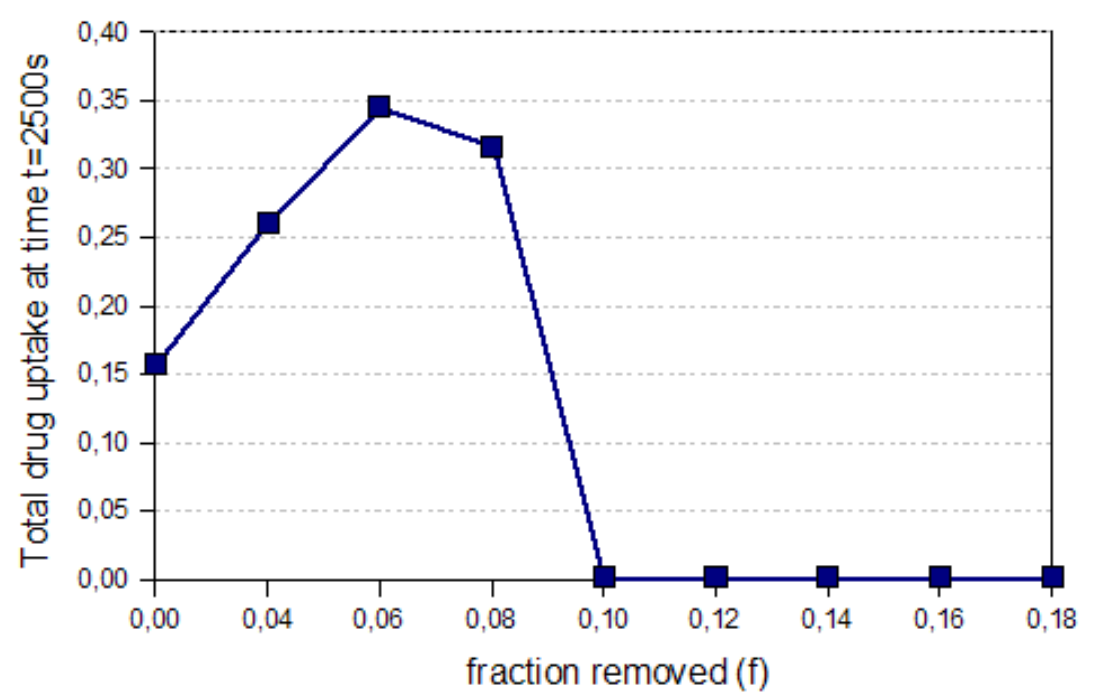

Figure 14: Plot of the total amount of drug received by the tumour at $t=2500 \mathrm{~s}$ vs. fraction of vessels randomly removed from the capillary bed (the fraction removed, FR, is varied from 0.0 to $0.18)$.

Influence of the fraction removed upon the drug uptake by the tum our

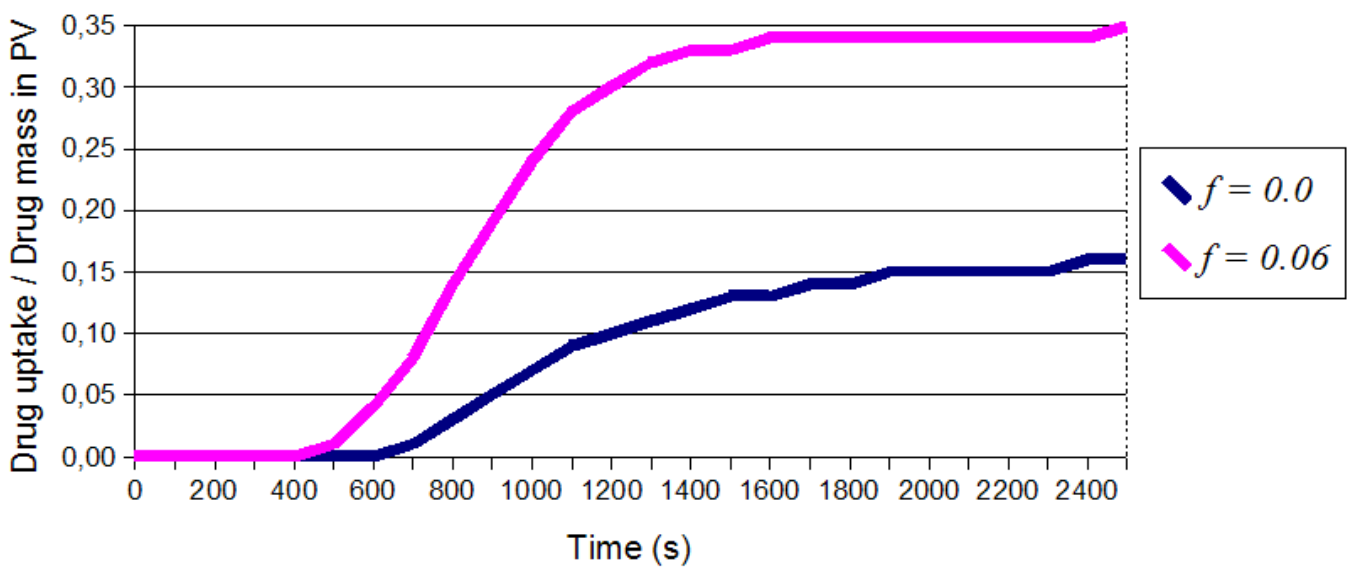

Figure 15: Plot showing the comparison of the drug uptake between the $f=0.06$ vasculature and the original $f=0.0$ case. 


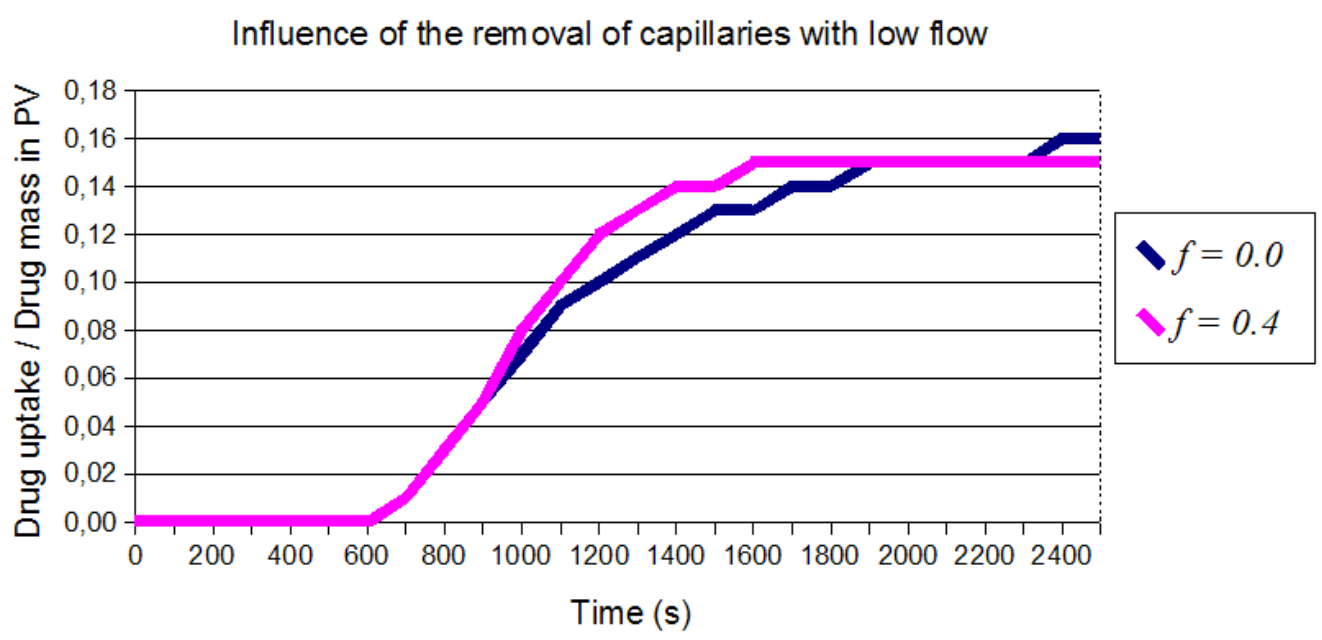

Figure 16: Plot showing the comparison of the drug uptake between the $f=0.4$ vasculature and the original $f=0.0$ case.

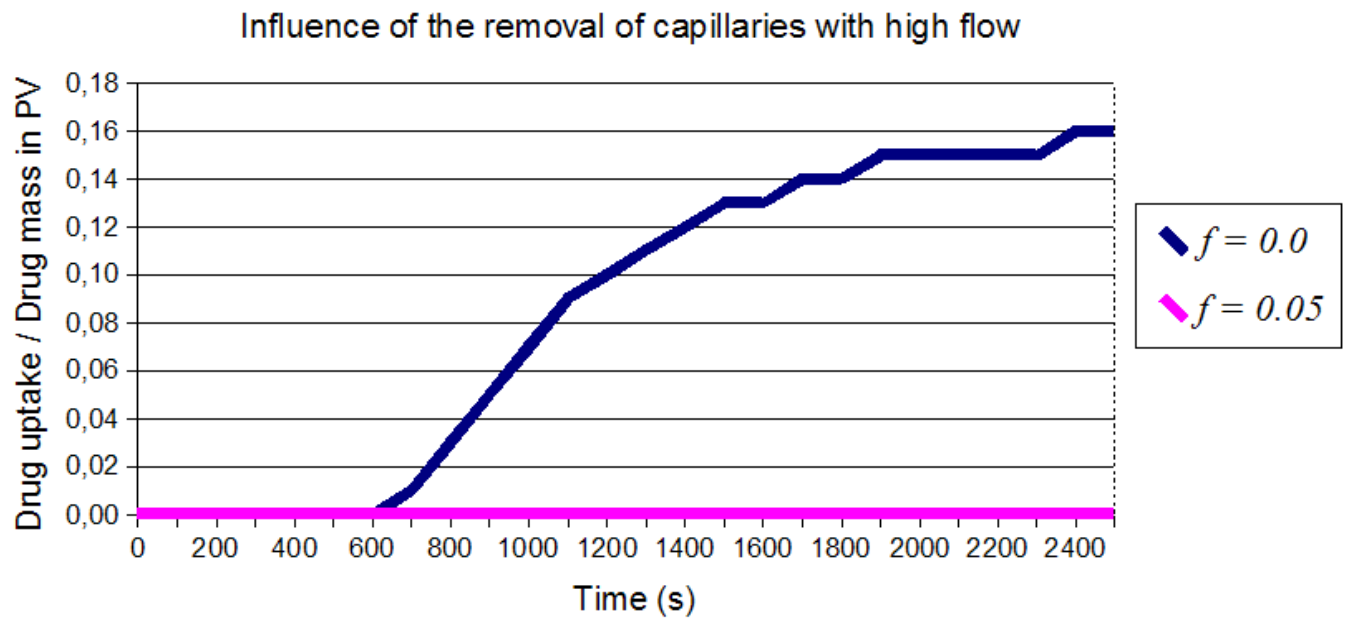

Figure 17: Plot showing the comparison of the drug uptake between the $f=0.05$ vasculature and the original $f=0.0$ case. 
from the recent literature that the stability of a developing capillary network — and therefore the efficacy of a vascular disrupting agent (VDA) - is closely linked to the corresponding pericyte coverage of the constituent capillaries. Moreover, pericytes are a prime target for new VDAs currently in clinical trials, and so any modelling framework aimed at capturing the main features of anti-vascular and anti-angiogenic treatments should somehow acknowledge pericyte-endothelium interactions during angiogenesis. It is the hoped that the current approach could ultimately be used to identify a VDA-specific "plasticity window" (i.e. a time period corresponding to low pericyte density), within which a given VDA would be most effective.

This section consequently begins by describing a simple model for pericyte recruitment during vessel maturation, whereby developing capillaries recruit stabilising pericytes from the surrounding tissue. Under physiological conditions, four main recruitment pathways have so far been identified [10] [23] (Chantrain et al, 2006, Jain, 2003): (i) latent TGF- $\beta 1$ secreted by endothelial cells, activated by proteases during angiogenesis, and bound to ALK5 receptors on neighbouring pericytes; (ii) sphingosine 1-phosphate (S1P) signalling from mast cells and platelets to receptor EDG-1 on endothelial cells, promoting N-cadherin trafficking and strengthening pericyte contacts; (iii) the Ang-1/Tie 2 signalling loop, whereby Tie 2 expressed by endothelium forma a complex with its agonistic ligand Ang- 1 expressed by perivascular cells; (iv) PDGF-B/PDGFR- $\beta$ signalling between sprouting endothelial cells and mural cells. The precise details of these various signalling cascades are only now being uncovered and some pathways appear to be interdependent. The most salient features of these mechanisms, however, can initially be distilled into a relatively small number of modelling assumptions.

As a first attempt towards capturing the process of pericyte recruitment, a simple logistic law is assigned to all capillary elements, allowing pericyte coverage to increase with time towards some limiting carrying capacity. The following relationship is used:

$$
\frac{d p}{d t}=\beta \cdot K(R) \cdot p\left(1-\frac{p}{K(R)}\right)
$$

where $p(t)$ denotes the total number of pericytes covering a vessel at time $t, \beta$ is a constant related to the degree of contact inhibition among pericytes, and $\mathrm{K}(\mathrm{R})$ is the carrying capacity of the vessel segment. The term $\beta \mathrm{uK}(\mathrm{R})$ is equivalent to an intrinsic recruitment rate for pericytes. Note that, in the base-case scenario, the carrying capacity is taken to depend upon vessel radius, more specifically:

$$
K(R)=\frac{\text { Surface Area of Vessel Segment }}{\text { Surface Area of Pericyte }}
$$

where the surface area of a pericyte is taken to be $\pi \mathrm{R}_{p c}^{2}$ with $\mathrm{R}_{p c}=20 \mu \mathrm{m}$. This, in effect, means that smaller capillaries have a smaller carrying capacity and recruit pericytes at a slower rate (due to fewer receptors/ligands being available on the vessel wall and a reduced surface area for expression of signalling molecules). Although the simplifying assumption has been made here that the entire capillary wall could become covered with pericytes (whereas, in reality, fenestrations between elongated mural cells are often in evidence), this could easily be modified by means of a constant in the equation defining $\mathrm{K}(\mathrm{R})$. However, this can be expected to have little effect upon the pericyte densities shown below. In all of the following simulations, the fully dynamic DATIA angiogenesis 
model was used to grow and remodel the capillary bed. Once again, only 2D results will be presented initially for ease of interpretation, with a few 3D comparisons shown at the end for completeness.

The base-case simulation is presented in Figure 18 and serves to highlight the utility of the modelling approach adopted. The figure shows both the developing capillary bed and the evolution of pericyte density (normalised to the carrying capacity of each capillary). Experimentally, the pericyte recruitment process occurs on the same timescale as the angiogenesis process but appears to lag the migration of endothelial cells by several days (Benjamin et al,. 1998). This is reproduced by the model over a wide range of $\beta$ values $(0.0075,0.075)$, with substantial pericyte coverage only occurring after approximately $\tau=4$ ( $\mathrm{t}=6.4$ days). In the base-case simulation, only flowing vessels were allowed to recruit pericytes, resulting in a highly heterogeneous spatial distribution of stabilised capillaries that corresponds broadly to the main flowing backbone of the vasculature. Notice however, that between times $\tau=11$ and $\tau=15$ some vessels dilate (branch 4 leaving the parent vessel, for example), whilst others constrict, and this impacts upon the density of the pericyte coating of these vessels - dilation of a vessel without additional recruitment effectively reduces its pericyte density (and stability), whilst a constricting vessel becomes more stable. Hence, although a vessel may initially appear functionally stable at some point during angiogenesis, subsequent flow-induced dilation may reveal a window of instability that would help VDA treatment. The results suggest that the plasticity window for this particular network is $\tau<11$ ( $\mathrm{t}<18$ days), as high pericyte densities are restricted to regions proximal to the parent vessel up to this point. Moreover, it is clear that treating this capillary bed at a later time with a VDA specific to endothelial cells (i.e. the blue regions) would be of only temporary benefit, as the main skeleton of enveloping pericytes would remain as a template for future endothelial migration. Conversely, the simulation shows that a VDA targeted at disrupting pericyte-endothelium adhesion could be more effective, starving the tumour of an efficient nutrient supply by removing the main flowing backbone of the network.

The second simulation, shown in Figure 19, corresponds to a tenfold increase in intrinsic recruitment rate $(\beta=0.075)$. Pericyte recruitment again lags endothelial cell migration by several days and the pericyte density distribution up to approximately $\tau=7$ is the same as that observed in the base case. However, increased recruitment ultimately results in a more stable network after approximately 18 days $(\tau=11)$. Furthermore, the increased pericyte coverage of even small capillaries means that this network would be difficult to eradicate even with a combination of endothelium-specific and pericyte-specific VDAs - although the high concentrations of VDA convected through the main backbone would expedite its removal, a widespread template of low-flow pericyte-covered capillaries would probably remain (relying on diffusion-driven mass transport from dilated vessels for treatment). This suggests that the treatment efficacy of any infused drug would generally depend upon a local combination of convective, diffusive, and reactive transport mechanisms (quantifiable in terms of local Peclet and Damköhler numbers).

The results of the previous two simulations highlight the importance of pericyte recruitment rate in determining the treatment potential of a given tumour-induced capillary bed. They demonstrate how the model can be used to suggest additional experimental studies in order to help justify or refute various model assumptions. One of the main assumptions of the modelling approach thus far has been the radius-dependency of the pericyte carrying capacity and Figure 20 shows the im- 

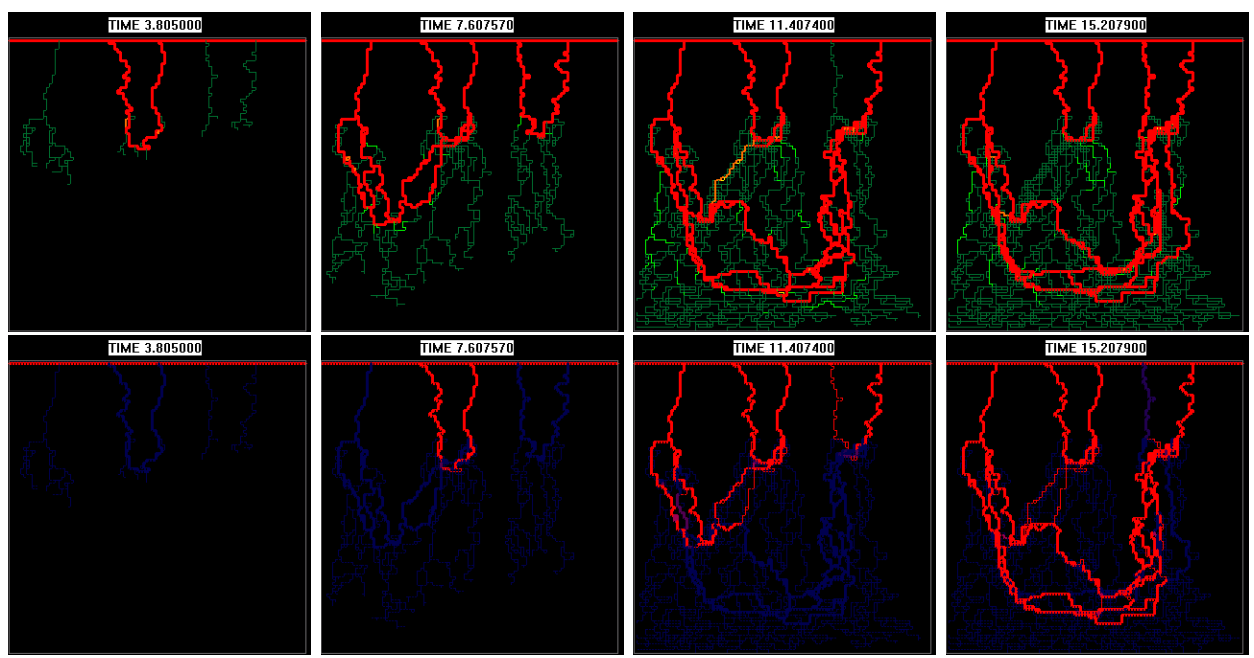

Figure 18: Snapshots of the developing capillary bed (top) and pericyte density (bottom) at different dimensionless times $(\tau=\mathrm{t}$ (days)/1.6). Only flowing vessels recruit pericytes and the recruitment parameter $\beta=0.0075$. For the pericyte figures, blue corresponds to low pericyte density and red corresponds to high pericyte density.
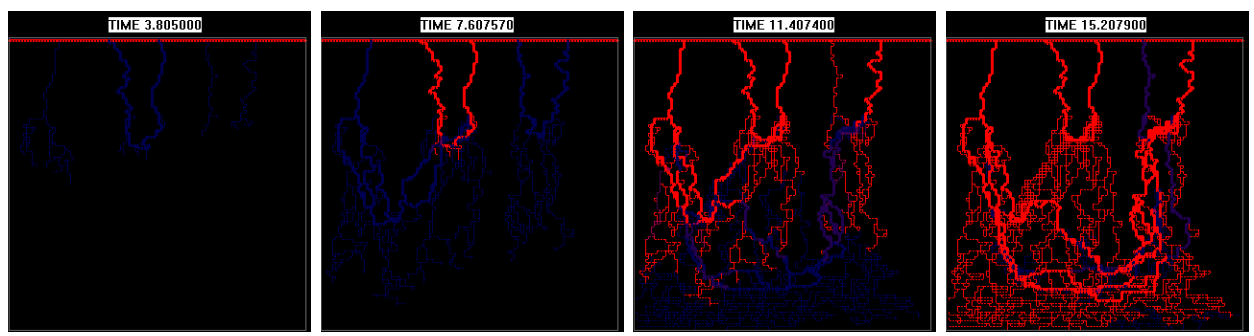

Figure 19: Snapshots of the pericyte density evolution at different dimensionless times $(\tau=$ $\mathrm{t}($ days $)$ /1.6). Again, only flowing vessels recruit pericytes but the recruitment parameter is increased tenfold $(\beta=0.075)$. Blue corresponds to low pericyte density and red corresponds to high pericyte density. 
pact of relaxing this by setting the carrying capacity of all vessels to be constant (taken to be that corresponding to a vessel of average radius, $7 \mu \mathrm{m}$ ). It is immediately clear that this has the effect of lengthening the treatment window considerably, as pericyte recruitment lags capillary migration by approximately 16 days and subsequent vessel dilation results in a low pericyte density around the main flowing backbone of the bed. Indeed, the low pericyte density is sustained indefinitely in these regions due to the limit imposed upon pericyte carrying capacity. Note also that the distribution of small, stable capillaries lies somewhere between those shown earlier when a varying carrying capacity was used (compare snapshots at $\tau=15$, for example, in Figures 18, 19, and 20). In the case of a fixed (average) carrying capacity, it would therefore appear that the main flowing backbone would remain highly susceptible to a VDA targeting pericytes for some considerable time. Although a considerable number of small pericyte-coated capillaries would again remain after treatment, these would be efficiently disconnected from the main parent vessel. Furthermore, if anti-endothelial treatment were supplied within the $\tau<15$ plasticity window, then these capillaries would also remain disconnected from the tumour surface and the surviving pericyte template would remain relatively ineffectual.
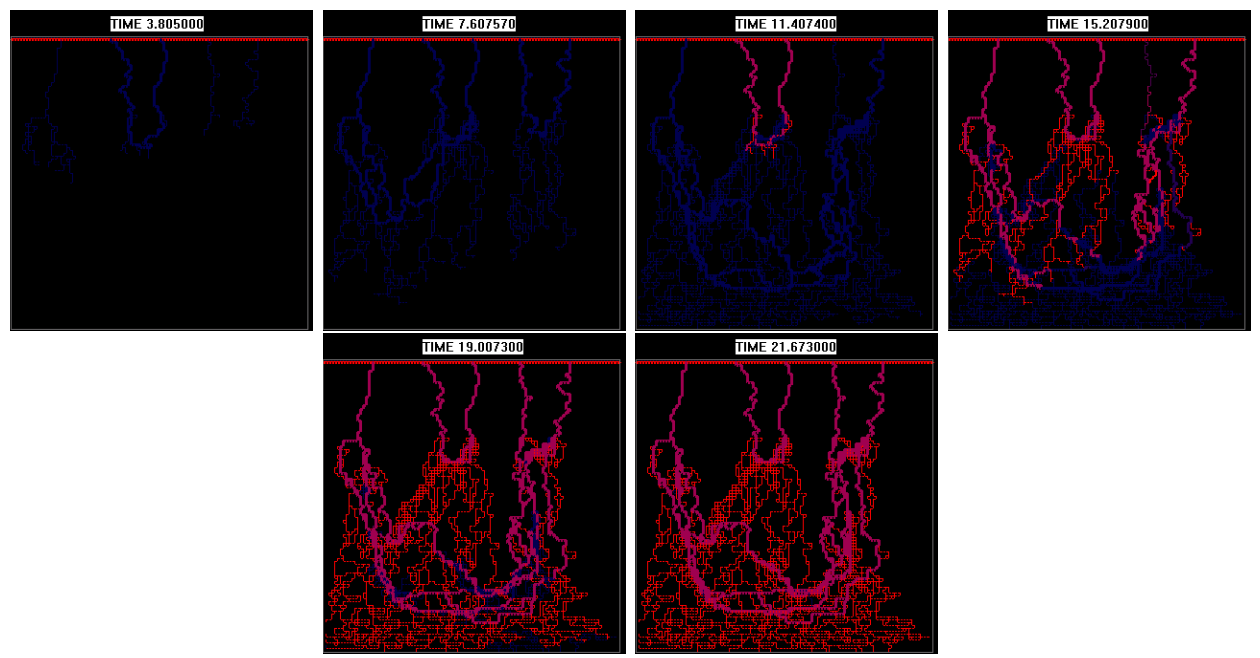

Figure 20: Snapshots of the pericyte density evolution at different dimensionless times $(\tau=$ $\mathrm{t}$ (days)/1.6). Again, only flowing vessels recruit pericytes but the carrying capacity is fixed at some average value (see text). $\beta=0.0075$. Blue corresponds to low pericyte density, magenta to intermediate density and red to high density.

Finally, two sensitivities are presented that include feedback between pericytes and endothelium by means of an explicit interdependency between vessel remodelling potential and local pericyte density. In this case, vessels could only remodel if their pericyte coverage was below $0.5 \mathrm{~K}_{\max }(\mathrm{R})$, where $\mathrm{K}_{\max }(\mathrm{R})$ corresponds to the radius-dependent maximum carrying capacity of a given vessel. This implies a correlation between network stability and pericyte coverage, which is thought to be the case physiologically [20] (Hughes et al, 2006). Figure 21(a) shows the pericyte coverage after approximately 24 days of migration and recruitment $(\tau=15)$ when only flowing vessels are able to recruit. The figure is similar to that shown for the base-case simulation with a few 
minor, but important, differences. The regression of two dilated vessels in the base-case (circled in Figure 21(c)) is no longer possible when the potential for capillary remodelling is coupled to pericyte density - the high pericyte coverage of these vessels at an earlier time means that they remain dilated for the remainder of the simulation. Note also, that the increased stability of the backbone in this area perturbs subsequent reinforcement of the network elsewhere, leading to a different downstream architecture (observe also that branch 4 (arrowed in Figure 21(c) is unable to dilate in response to remodelling cues, unlike the base-case). Such minor changes in bed architecture can have a significant impact upon transport issues, causing increased bypassing of injected chemotherapy treatments. The modelling results suggest that it is important to determine the degree to which vascular remodelling can occur as a function of pericyte density in order to optimise such therapies. The final simulation was undertaken to examine the assumption that only flowing vessels recruit pericytes. The initial assumption was based on experimental observations that show higher pericyte densities associated with more mature capillary structures in flowing retinal vasculature. However, reliable fate mapping of pericytes in vivo is not possible at present [3] (Armulik et al, 2005) and pericyte recruitment by tumour-induced vasculatures is not well understood. In light of these uncertainties, a simulation was run assuming that all nascent capillaries could recruit pericytes from the surrounding tissue, whether flowing or not. Once again, vessels could only remodel if their pericyte coverage was below $0.5 \mathrm{~K}_{\max }(\mathrm{R})$. The result is shown in Figure $21(\mathrm{~b})$, and shows a very different structure from those seen previously. In this case, only the primary anastomosis has had time to dilate before pericyte density restricts all subsequent remodelling throughout the migrating bed. This type of bed would be a worst case scenario for VDA treatment, as numerous small, low-flow capillaries would need to be accessed by the injected drug. Most of the treatment would simply be recirculated to the parent vessel, however, by way of the single dilated arcade.

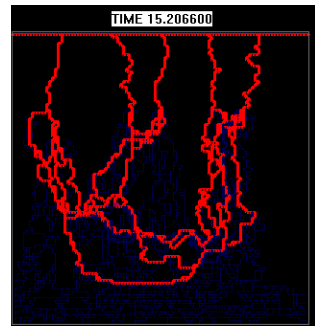

(a)

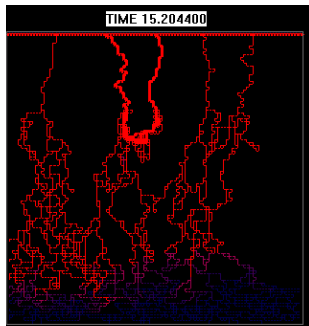

(b)

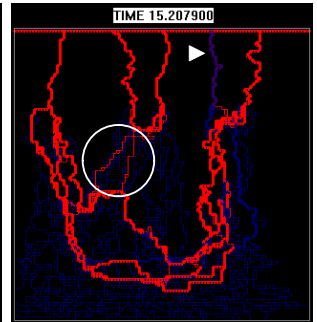

(c)

Figure 21: Snapshots of the pericyte density evolution at different dimensionless times $(\tau=$ $\mathrm{t}($ days $) / 1.6)$ where vessels can only remodel if pericyte coverage is less than $0.5 \mathrm{~K}_{\max }(\mathrm{R})$. (a) only flowing vessels recruit; (b) all vessels recruit; (c) base case simulation for comparison. $\beta=0.0075$. Blue corresponds to low pericyte density, magenta to intermediate density and red to high density.

All of the pericyte recruitment simulations shown thus far have been undertaken in two dimensions in order to aid interpretation. However, it has already been shown that quantitative predictions of flow-related delivery require 3D networks. As the future aim of the modelling framework developed here will be to compare numerical results with experimental VDA delivery data, it is important to extend the pericyte work into the third dimension. Hence, this section concludes by 
presenting two fully adaptive, three-dimensional angiogenesis simulations that incorporate pericyte recruitment. Figure 22(a-c) shows the three-dimensional equivalent of the base-case simulation in Figure 18, whilst Figure 22(d-f) shows the case when a fixed carrying capacity is assumed for all vessels. Heterogeneity in pericyte coverage is evident in both cases and pericyte coverage again lags endothelial migration by several days. These figures clearly highlight the difficulties faced by researchers in developing generic VDAs aimed at disrupting such heterogeneous aberrant vasculatures.

Of course, many more sensitivities could be examined using the modelling framework described here but the main purpose has been to demonstrate the need for additional experimental observations to clarify the main mechanisms affecting pericyte recruitment and distribution. Armed with this knowledge, it should be possible to optimise the potential of anti-angiogenic and anti-vascular drugs by coupling modelling and experiment.

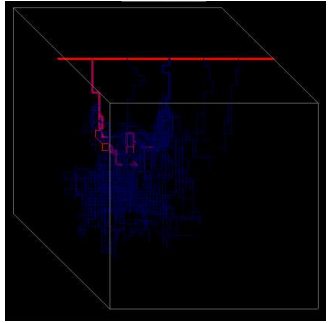

(a)

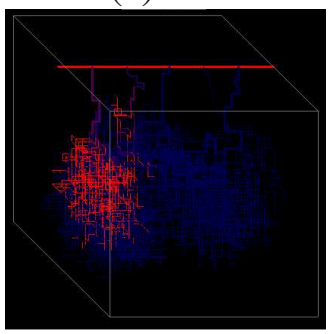

(d)

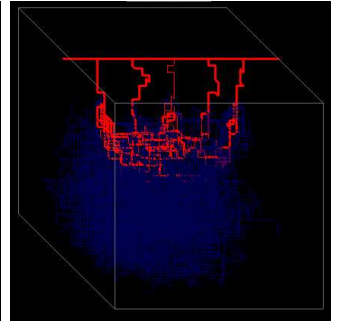

(b)

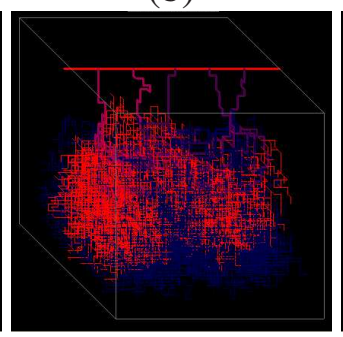

(e)

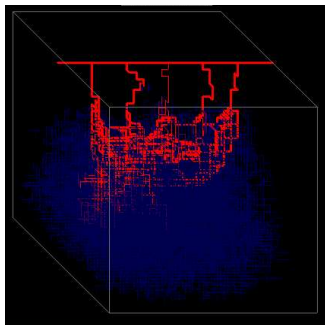

(c)

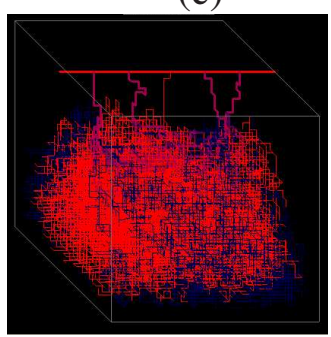

(f)

Figure 22: Snapshots of the 3D pericyte density evolution at different dimensionless times $(\tau=$ $\mathrm{t}($ days)/1.6). (a-c) base case simulation at $\tau=7, \tau=10, \tau=12$ (see text); (d-f) fixed average carrying capacity at $\tau=8, \tau=10, \tau=12$. $\beta=0.0075$. Blue corresponds to low pericyte density, magenta to intermediate density and red to high density.

\section{Discussion and Conclusions}

This paper has presented a number of results primarily related to convective transport through tumour-induced angiogenic capillary beds. Whilst there have been many modelling studies reported in the literature dealing with the process of angiogenesis itself - i.e. the way in which the new capillary bed is initiated and migrates - there have been relatively few studies examining the important role played by blood perfusion during migration and fewer still examining the delivery 
of chemotherapeutic compounds through aberrant tumour vasculature. In response to the paucity of transport models in this area, a new framework has been developed within which a wide range of therapeutic interventions can be studied.

Although the salient features of the extended angiogenesis model have been described, including the effects of matrix degrading enzymes and perfusion-related capillary remodelling, the main aim of the paper has been to study the way in which bed architecture and spatial heterogeneity affect the evolution of intravenous/intra-arterial drug treatments. Initially, two- and threedimensional simulations of chemotherapeutic delivery to the tumour periphery via both static and dynamically adaptive networks were compared. It was demonstrated that, instead of supplying drug to the tumour, some branches of the induced vasculature actually remove drug from the network, thereby reducing its efficacy. Indeed, results from a 30-second bolus injection simulation into a network formed in response to a focussed semicircular TAF source highlighted the possibility of the treatment bypassing the tumour altogether.

Simulations using the adaptive model under different parameter regimes highlighted a number of new therapeutic targets for tumour management. For example, reduction of the haptotactic response of the migrating endothelial cells during angiogenesis was shown to reduce lateral migration of the vessels and reduce shear-induced branching. Subsequent evolution of blood perfusion through this network suggested that tumours supplied by this type of vasculature would be wellsupplied with nutrients and could be expected to grow rapidly. However, such tumours would also be highly susceptible to infused treatments, with cytotoxic agents reaching the tumour surface in higher concentrations than the base-case simulation. A second set of simulations suggested that a depressed haematocrit could be expected to lead to the formation of highly dilated arcades close to the parent vessel. Although this had the positive effect of causing more drug to enter the capillary network than entered in the base-case simulation, delivery to the tumour was reduced by more than three orders of magnitude. This is a rather negative result in the context of infused treatment, but it also suggests that decreasing local haematocrit could be a possible mechanism for generating vasculatures that are detrimental to further tumour growth.

The therapeutic applications described in the earlier part of the paper focussed upon the impact of bed topology on the delivery of a cytotoxic drug from parent vessel to tumour periphery. However, these simulations largely neglected the important possibility of disrupting the vascular network itself. As new treatments are being tailored to specifically target vascular endothelium, it was decided to examine the effects of anti-angiogenic and anti-vascular therapies specifically aimed at disrupting the vascular bed. These therapies were initially modelled by means of simple "capillary pruning" algorithms that were used to modify the network connectivity in a number of different ways. It was found that random removal of vessels (approximating the action of antivascular drugs) led to a significant increase in the amount of drug delivered to the tumour, whilst selective removal of vessels characterised by low flow (used to mimic anti-angiogenic treatment) was seen to accelerate delivery by approximately $30 \%$. The possibility of targeting "bottleneck" capillaries, characterized by high wall stresses, was also investigated and the network was essentially shut down in this case. Hence, if a treatment could be developed to target these fast-flowing "bottleneck" capillaries, it would be a highly efficient way of slowing tumour growth.

Of course the capillary pruning approach is rather unsophisticated and a number of the under- 
lying biological principles have been subsumed into very basic algorithms. Although the approach broadly captured the effects of vascular disrupting agents (VDAs), it is clear from the recent literature that the stability of a developing capillary network — and therefore the efficacy of a VDA — is closely linked to the corresponding pericyte coverage of the constituent capillaries. Hence, in order to study the effects of different anti-vascular and anti-angiogenic therapies more rigorously, a new model was required that explicitly incorporated the important angiogenic mechanism of pericyte recruitment. As a first step towards capturing this process, a simple logistic law was assigned to all capillary elements, allowing pericyte coverage to increase with time towards some limiting carrying capacity. The spatial distribution of pericyte density was investigated in both 2D and 3D under a number of different model assumptions and the implications for anti-angiogenic and anti-vascular treatments were discussed. It was found that the recruitment of pericytes lagged the migration of endothelial cells by several days over a wide range of intrinsic recruitment rates $(0.0075,0.075)$, in agreement with experimental observations [7] (Benjamin et al,. 1998). Moreover, it was shown that, although a vessel may initially appear functionally stable at some point during angiogenesis, subsequent flow-induced dilation may reveal a window of instability that would help VDA treatment. However, although high concentrations of VDA were observed flowing through the main backbone of all capillary networks studied (thereby expediting its removal), a widespread template of low-flow pericyte-covered capillaries was left behind. Treatment of this pericyte template would rely on diffusion-driven mass transport from dilated vessels and this suggests that the treatment efficacy of any infused drug would generally depend upon a local combination of convective, diffusive, and reactive transport mechanisms (quantifiable in terms of local Peclet and Damköhler numbers). A more detailed study of this issue will form the basis of future simulation work and it is hoped that the modelling framework could ultimately be used to identify a VDA-specific "plasticity window" (i.e. a time period corresponding to low pericyte density), within which a given VDA would be most effective.

Throughout this paper, both two- and three-dimensional simulation results have been presented and it has been shown that extending the model to three dimensions reduces chemotherapy delivery to a tumour periphery by an order of magnitude. These results clearly demonstrated that, although two-dimensional studies are valuable in a qualitative sense, three dimensional angiogenesis modelling appears to be a necessary prerequisite for quantitative prediction for comparison with in vivo results. Of course, many more sensitivities could be examined using the framework described here but one of the main aims has been to demonstrate the need for additional experimental observations to clarify a number of outstanding issues. For example, the key mechanisms governing pericyte recruitment and drug uptake require additional research, as do the metabolic stimuli affecting blood vessel dynamics.

Armed with this adjuvant knowledge, it should be possible to refine the model still further, perhaps by including discrete pericyte migration and improved dose-response algorithms. By closely coupling an improved mathematical framework with corresponding laboratory experiments, it should prove possible to increase the potential of a range of anti-angiogenic and anti-vascular drugs in the future. 


\section{Acknowledgments}

MAJC and SMcD gratefully acknowledge the support of BBSRC Grant BBF0022541 "Guidance cues and pattern prediction in the developing retinal vasculature: a combined experimental and theoretical modelling approach". The work of MAJC was also supported by a European Research Council Advanced Investigator Grant, No. 227619, "From Mutations to Metastases: Multiscale Mathematical Modelling of Cancer Growth and Spread".

\section{Appendix A: Capillary Growth Details}

In the earlier model of Anderson and Chaplain (1998) [2], endothelial cell densities and their global influence on TAF and FN concentrations were considered in a continuous formulation. Here, the focus is on local effects and so the influence of each individual cell on its local environment is considered. In order to achieve this, the displacement of each individual endothelial cell, located at the tips of growing sprouts, is given by the discretised form of the endothelial cell mass conservation equation (2.1).

The chemotactic migration is characterised by the function $\chi(c)=\chi /(1+\delta c)$, which reflects the decrease in chemotactic sensitivity with increased TAF concentration. The coefficients $D, \chi$ and $\rho$ characterise the random, chemotactic and haptotactic cell migration respectively (full details of the nondimensionalisation can be found in [2] Anderson and Chaplain, 1998). The migration of each cell in 2D is consequently determined by a set of normalized coefficients emerging from this equation (Figure A1), which relate to the likelihood of the cell remaining stationary $\left(\mathrm{P}_{0}\right)$, or moving left $\left(\mathrm{P}_{1}\right)$, right $\left(\mathrm{P}_{2}\right)$, up $\left(\mathrm{P}_{3}\right)$ or down $\left(\mathrm{P}_{4}\right)$ :

$$
n_{l, m}^{q+1}=n_{l, m}^{q} P_{0}+n_{l+1, m}^{q} P_{1}+n_{l-1, m}^{q} P_{2}+n_{l, m+1}^{q} P_{3}+n_{l, m-1}^{q} P_{4}
$$

where $l$ and $m$ are positive parameters which specify the position of the endothelial cell on the 2D spatial grid i.e. $x=l \Delta x$ and $y=l \Delta y$ and time discretisation is represented by $t=q \Delta t$. These coefficients $P_{0}-P_{4}$ incorporate the effects of random, chemotactic and haptotactic movement and depend upon the local chemical environment (FN and TAF concentrations). In 3D, the discretised endothelial equation has the form:

$$
\begin{aligned}
n_{l, m, w}^{q+1}= & n_{l, m, w}^{q} P_{0}+n_{l+1, m, w}^{q} P_{1}+n_{l-1, m, w}^{q} P_{2}+n_{l, m+1, w}^{q} P_{3}+ \\
& n_{l, m-1, w}^{q} P_{4}+n_{l, m, w+1}^{q} P_{5}+n_{l, m, w-1}^{q} P_{6} .
\end{aligned}
$$

With additional movement coefficients: in $\left(\mathrm{P}_{5}\right)$ or out $\left(\mathrm{P}_{6}\right)$ The model is then given by the following set of equations:

$$
\frac{\partial n}{\partial t}=\overbrace{D \nabla^{2} n}^{\text {random }}-\overbrace{\nabla \cdot(\chi(c) n \nabla c)}^{\text {chemotaxis }}-\overbrace{\rho \nabla \cdot(n \nabla f)}^{\text {haptotaxis }},
$$




$$
\begin{aligned}
\frac{\partial c}{\partial t} & =-\eta n_{k} c, \\
\frac{\partial f}{\partial t} & =\beta n_{k}-\gamma m f \\
\frac{\partial m}{\partial t} & =\alpha n_{k}+\varepsilon \nabla^{2} m-\nu m,
\end{aligned}
$$

where $c$ represents the TAF concentration, $f$ the FN concentration, $m$ the MDE density and $n_{k}$ a Boolean value ( 1 or 0 ) that indicates the presence or absence of an endothelial cell at a given position. The parameters $\beta$ and $\alpha$ characterise the production rate by an individual endothelial cell of FN and MDE respectively and $\eta$ its TAF consumption rate. The major difference with the earlier model is that degradation of fibronectin $f$, characterised by the coefficient $\gamma$, no longer depends directly on the endothelial cell density $n$. This now depends upon the MDE density $m$ produced by each individual endothelial cell $n_{k}$ at rate $\alpha$. The MDF once produced, diffuses locally with diffusion coefficient $\varepsilon$, and is spontaneously degraded at a rate $\nu$.

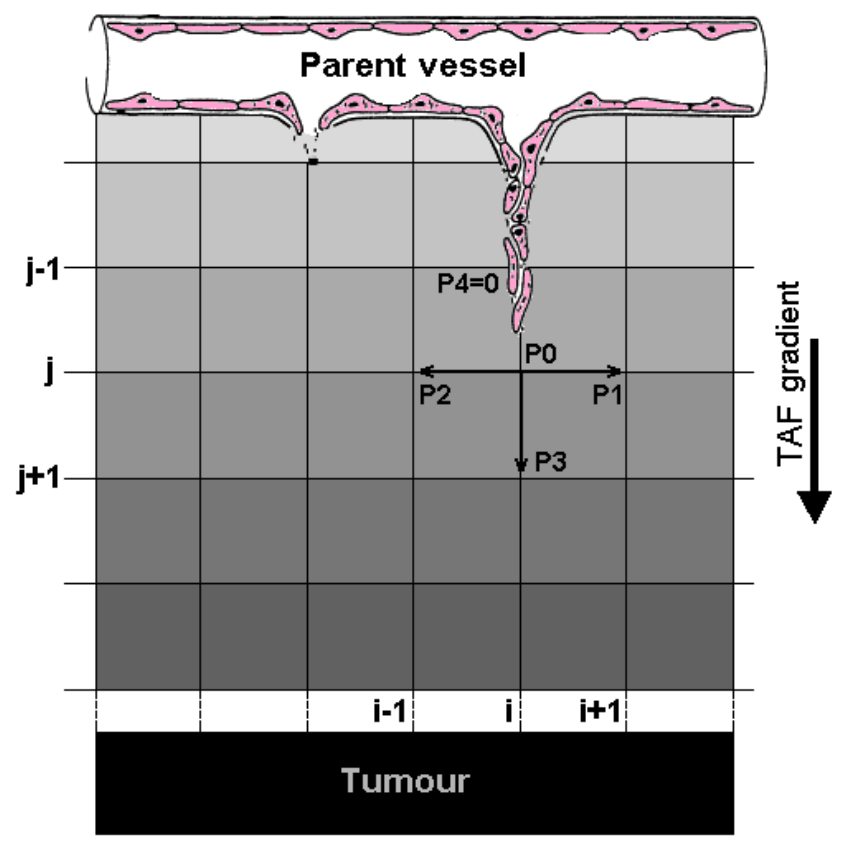

Figure 23: Schematic diagram of a section of the 2D-grid used in the numerical computation procedure illustrating how the process of the migration of tip endothelial cells, and hence capillary sprout growth, is taken into account in the simulations. At each node, the sprout tip can grow in 3 possible directions in 2D (and 5 possible directions in 3D).

In earlier work [2] [28] [45] [46] (Anderson and Chaplain, 1998; McDougall et al (2002), Stéphanou et al. (2005a, b)), branching at the capillary tips was assumed to depend only upon 
the local TAF concentration. This formulation has been used again here and the corresponding tip branching probabilities are shown in Table 1. In addition to tip branching, however, the physiologically significant process of vessel branching is also modelled as part of the current study. In order to implement this effect in the model, we assume that branching along a vessel (i.e. the generation of a new vessel which branches out at some point along an existing vessel wall as distinct from the vessel tip) depends both on the TAF concentration and on the wall shear stress (WSS). Table 2 shows the dependence of vessel branching probability as a function of the combined effects of local wall shear stress and local TAF concentration.

\begin{tabular}{|l|l|}
\hline $\begin{array}{l}\text { TAF Concentra- } \\
\text { tion }\end{array}$ & Sprout Tip Branching Probability \\
\hline$\leq \quad 0.3$ & 0.0 \\
\hline$] 0.3-0.5]$ & 0.2 \\
\hline$] 0.5-0.7]$ & 0.3 \\
\hline$] 0.7-0.8]$ & 0.4 \\
\hline$>0.8$ & 1.0 \\
\hline
\end{tabular}

Table 1: Sprout tip branching probabilities as a function of the local TAF concentration

\begin{tabular}{|c|c|c|c|c|c|c|}
\hline & \multicolumn{5}{|c|}{$\mathbf{W S S} / \tau_{\max }$} \\
\hline & & {$[0.0,0.2[$} & $\overline{\Gamma 0.2,0.4[}$ & {$[0.4,0.6[$} & $\overline{[0.6,0.8[}$ & {$[0.8,1.0]$} \\
\hline \multirow{5}{*}[\mathrm{TAF}]{$/ \mathrm{TAF}_{\max }$} & {$[0,0,0.3[$} & 0.00 & 0.00 & 0.00 & 0.00 & 0.00 \\
\hline & {$[0.3,0.5]$} & 0.00 & 0.02 & 0.04 & 0.06 & 0.08 \\
\hline & {$[0.5,0.7[$} & 0.00 & 0.03 & 0.06 & 0.09 & 0.12 \\
\hline & {$[0.7,0.8[$} & 0.00 & 0.04 & 0.08 & 0.12 & 0.16 \\
\hline & {$[0.8,1.0]$} & 0.00 & 0.10 & 0.20 & 0.30 & 0.40 \\
\hline
\end{tabular}

Table 2: Vessel branching probabilities as functions of the local TAF concentration and the magnitude of the local wall shear stress. $\mathrm{TAF}_{\max }$ is the maximum TAF concentration at $\mathrm{t}=0$ and $\tau_{\text {max }}=2 \mathrm{~Pa}\left(20 \mathrm{dynes} / \mathrm{cm}^{2}\right)$, the maximum shear stress derived from preliminary flow simulations.

One additional constraint on vessel branching is the age of the vessel itself. The time interval within which a vessel can branch has been fixed at [4-8] days in the simulations (i.e. from $\tilde{\tau}=2.66$ to $\tilde{\tau}=5.33$ ). In this interval, the vessel is sufficiently mature for branching to occur yet young enough to ensure that no basal lamina has had time to form (which would contribute considerably to the stabilization of the network; [7] [32] Benjamin et al. 1998; Morikawa et al. 2002).

\section{Initial Conditions}

The domain considered for the computational simulation studies is a square of length $L=2 \mathrm{~mm}$ (2D) or a cube of edge length $0.6 \mathrm{~mm}$ (3D) and the parent vessel from which the vascular network grows 
is located at the upper edge/face of the domain. The tumour surface is located along the lower domain boundary. We assume that the capillary sprouts, TAF and MDE remain confined within the domain and so no-flux boundary conditions are imposed on the boundaries. Initial TAF and fibronectin profiles are the same as those used in [28] McDougall et al (2002), there is initially no MDE present and vascular growth is initialised by distributing five sprouts at regular intervals along the parent vessel.

\section{Cell migration parameters}

Unless otherwise indicated, the dimensionless parameter values used for the simulations presented in this paper were as follows [2] [28] [45] [46] (Anderson and Chaplain, 1998; McDougall et al., 2002; Stéphanou et al., 2005a,b):

$$
D=0.00035, \delta=0.6, \chi=0.38 \rho=0.16, \eta=0.1, \beta=0.05, \gamma=0.1, \alpha=10 \mathrm{e}-6, \varepsilon=0.01, \nu=3 .
$$

Capillary migration time was scaled as $\tilde{t}=\frac{t}{\tau}$ : with $\tau=L^{2} / D_{c}$, where $L=2 \mathrm{~mm}$ (2D) or $0.6 \mathrm{~mm}$ (3D) was the length of the domain and $D_{c}=2.9 \times 10^{-7} \mathrm{~cm}^{2} s^{-1}$ was taken as the diffusion coefficient for TAF (Bray, 1992). We note that the timescale associated with the capillary growth process is of the order several days, whereas flow through the parent vessel and capillary network occurs over a timescale of a few seconds to a few minutes. A discussion of how the two timescales are coupled in the full adaptive model is given in [29] McDougall et al (2006).

\section{Appendix B: Adaptive Flow Modelling Details}

\section{General Model}

In its most general form, the flow model developed here consists of a three-dimensional cubic network of bond elements. These bonds can be thought of as straight cylindrical capillary elements, although the constraint of cylindrical geometry can easily be relaxed. Now, for a single capillary element $\mathrm{i}$ of radius $\mathrm{R}_{i}$ and length $\mathrm{L}_{i}$, the elemental fluid flow rate in the capillary is given by Poiseuille's law:

$$
Q_{i}=\frac{\pi R_{i}^{4} \Delta P_{i}}{8 \mu L_{i}}
$$

where $\mu$ is the fluid viscosity and $\Delta \mathrm{P}_{i}$ the pressure drop across the element. At each node (junction), six elements meet (in 3D) and (assuming incompressible flow) mass conservation means that the sum of all six flows must add up to zero, i.e.

$$
\sum_{i=1}^{i=6} Q_{i}=0
$$

Consideration of the whole network then leads to a set of linear pressure equations, the solutions to which (using e.g. Successive Over-Relaxation (SOR), Choleski conjugate gradient method, Lanczos method) can then be used to calculate elemental flows. 


\section{Blood Rheology}

When dealing with a non-Newtonian fluid, such as blood, the flow-pressure drop relationship can be approximated by the following Poiseuille-like expression:

$$
Q=\frac{\pi R^{4} \Delta P}{8 \mu_{a p p}\left(R, H_{D}\right) L}
$$

where $\mu_{a p p}\left(R, H_{D}\right)=\mu_{\text {rel }} \times \mu_{\text {plasma }}$ is the apparent blood viscosity, which depends upon the local blood haematocrit, the radius of the vessel through which the blood is flowing, and the underlying plasma viscosity, $\mu_{\text {plasma }}$. (See Equations (2.3) and (2.4) in main text).

\section{Vessel Adaptation}

In this paper, vessel adaptation follows the treatment of [35, 36, 37, 38] Pries et al. $(1995,1998$, 2001a). The model considers a number of stimuli affecting vessel diameter that account for the influence of the wall shear stress $\left(S_{w s s}\right)$, the intravascular pressure $\left(S_{p}\right)$, and a metabolic mechanism depending on the blood haematocrit $\left(S_{m}\right)$. These stimuli form a basic set of requirements in order to obtain stable network structures with realistic distributions of vessels diameters and flow velocities. A brief description of each now follows.

(i) Wall shear stress - Many studies show that vessels adapt their radius in order to maintain a constant level of wall shear stress [36] [37] [38] [16] (Pries et al. 1998, 2001a,b; Fung, 1993). Hence vessel radius tends to increase with increasing wall shear stress, whilst wall shear stress decreases with increasing radius. The wall shear stress stimulus can be described by a logarithmic law as

$$
S_{w s s}=\log \left(\tau_{w}+\tau_{r e f}\right)
$$

where $\tau_{w}$ is the actual wall shear stress in a vessel segment calculated from

$$
\tau_{w}=\frac{4 \mu\left(R, H_{D}\right)}{\pi R^{3}}|Q|
$$

and $\tau_{r e f}$ is a constant included to avoid singular behaviour at low shear rates [37] (Pries 2001a). Stresses in (B4) and (B5) are in dynes $/ \mathrm{cm}^{2}$. The wall shear stress calculated in the parent vessel of our computational model is of the order $4 \mathrm{~Pa}\left(40 \mathrm{dynes} / \mathrm{cm}^{2}\right)$ and capillary values are less than $2 \mathrm{~Pa}\left(20\right.$ dynes $\left./ \mathrm{cm}^{2}\right)$, in agreement with those measured experimentally in the dog by Kamiya et al. (1984) [24]. Adaptation in response to the wall shear stress stimulus alone tends to reinforce a single path in the network composed of a few well-established fully-dilated vessels — corresponding to the main flowing "backbone" of the vasculature - whilst simultaneously eliminating the lowflow paths. However, the resulting network is "unstable" in the sense that there is no consistent balance for the radius and flow distribution achieved when $S_{w s s}$ is considered in isolation.

(ii) Intravascular pressure - Intravascular pressure is another key stimulus for vascular adaptation. Pries et al. (1995) have experimentally observed on the rat mesentery the dependence of the magnitude of the wall shear stress with the local intravascular pressure $(P)$. They proposed a 
parametric description of their experimental data, which exhibits a sigmoidal increase of the wall shear stress with increasing pressure through the following:

$$
\tau_{e}(P)=100-86 \cdot \exp \left\{-5000 \cdot[\log (\log P)]^{5.4}\right\}
$$

Pressure is measured in $\mathrm{mm}$ of mercury $(1 \mathrm{mmHg}=133 \mathrm{~Pa})$ and stresses are again given in dynes $/ \mathrm{cm}^{2}$. The sensitivity of the corresponding stimulus to intravascular pressure is then described by:

$$
S_{p}=-k_{P} \log \tau_{e}(P)
$$

where $k_{P}$ is a constant that dictates the relative intensity of the stimulus.

(iii) Metabolic haematocrit-related stimulus - The metabolic stimulus effectively stabilises the adapting network by stimulating vessel growth in areas of the vascular bed exhibiting low flow. The stimulus is once again described by a logarithmic law and takes the form:

$$
S_{m}=k_{m} \log \left(\frac{Q_{r e f}}{Q H_{D}}+1\right)
$$

where $Q_{\text {ref }}$ is a reference flow. In our simulations, $Q_{\text {ref }}$ corresponds to the flow in the parent vessel. $H_{D}$ represents the discharge haematocrit in the vessels, $Q$ the flow in the vessel under consideration and $k_{m}$ is a constant characterizing the relative intensity of the metabolic stimulus.

Our theoretical model for vessel adaptation assumes that the change in a flowing vessel radius $(\Delta R)$ over a time step $\Delta t$ is proportional to both the global stimulus acting on the vessel and to the initial vessel radius $R$, i.e.

$$
\Delta R=S_{t o t} R \Delta t=\left(S_{w s s}+S_{p}+S_{m}\right) R \Delta t
$$

which leads to Equation (2.5) in the main text. Note that the additional term $k_{s}$ represents the shrinking tendency of a vessel. This term is interpreted by Pries et al. (1998) [36] as reflecting a natural reaction of the basal lamina, which acts to counter any increase in vessel diameter.

\section{Flow model parameters}

(i) Vessel Properties: For the a posteriori remodelling simulations presented, the initial radius of each capillary segment was taken to be $6 \mu \mathrm{m}$ and remodelling of the vessels was permitted within a range, from a minimum radius of $2 \mu m$ (essentially eliminating flow) to a maximum radius of $12 \mu \mathrm{m}$. During the DATIA simulations, nascent, non-flowing capillaries (i.e. those not yet part of the connected flowing network) were assigned $6 \mu \mathrm{m}$ radii and remodelling was again considered in the range $(2,12) \mu m$. In all simulations, the radius of the parent vessel was kept fixed at $14 \mu m$. These values correspond to vessel radii at the capillary level, where the size of the vessels are very close to the size of the red blood cells [12] (Ciofalo et al. 1999).

(ii) Adaptation Parameters: The parameters used for the base case adaptation model presented in Equation (2.5) were taken to be: 


$$
k_{s}=0.35, k_{p}=0.1, k_{m}=0.07, \tau_{\text {ref }}=0.103, Q_{\text {ref }}=1.909 e^{-11},
$$

(after [45] [46] Stéphanou et al., 2005a,b), where $Q_{\text {ref }}$ corresponds to the flow in the parent vessel, calculated from Equation (B3) with $R=14 \mu \mathrm{m}, L=2 \mathrm{~mm}$ and $\Delta \mathrm{P}=1200 \mathrm{~Pa}(9 \mathrm{mmHg}$ ) (the pressure drop across the parent vessel). The plasma viscosity $\mu_{\text {plasma }}$ is $1.2 \times 10^{-3} \mathrm{~Pa}$.s and this parameterisation gives perfusion velocities in the parent vessel of approximately $3 \mathrm{~mm} . \mathrm{s}^{-1}$. One of the main determinants of the extent of vascular remodelling is the intravascular pressure $(P)$. In the simulations carried out here, we have chosen inlet and outlet pressures to ensure average intravascular pressures of approximately $20 \mathrm{mmHg}$, in accordance with physiological values at the capillary scale.

\section{References}

[1] T. Alarcon, H.M. Byrne, P.K. Maini. A cellular automaton model for tumour growth in inhomogeneous environment. J. Theor. Biol., 225 (2003), 257-274.

[2] A.R.A. Anderson, M.A.J. Chaplain. Continuous and discrete mathematical models of tumorinduced angiogenesis. Bull. Math. Biol., 60 (1998), 857-899.

[3] A. Armulik, A. Abramsson, C. Betsholtz. (2005). Endothelial/pericyte interactions. Circulation Research, 97 (2005), 512-523.

[4] D.H. Ausprunk, J. Folkman. Migration and proliferation of endothelial cells in preformed and newly formed blood vessels during tumour angiogenesis. Microvasc. Res., 14 (1977), 53-65.

[5] R.G. Bagley. Pericytes from human non-small cell lung carcinomas: An attractive target for anti-angiogenic therapy. Microvascular Res., 71 (2006), 163-174.

[6] J.W. Baish, Y. Gazit, D.A. Berk, M. Nozue, L.T. Baxter, R.K. Jain. Role of tumor vascular architecture in nutrient and drug delivery: an invasion percolation-based network model. Microvasc. Res., 51 (1996), 327-346.

[7] L.E. Benjamin, I. Hemo, E. Keshet. A plasticity window for blood vessel remodelling is defined by pericyte coverage of the preformed endothelial network and is regulated by PDGF-B and VEGF. Development, 125 (1998), 1591-1598.

[8] D. Bray. Cell Movements. New-York: Garland Publishing, 1992.

[9] R.A. Brekken, P.E. Thorpe. Vascular endothelial growth factor and vascular targeting of solid tumors. 21 (2001), 4221-4229.

[10] C.F. Chantrain, P. Henriet, S. Jodele, H. Emonard, O. Feron, P.J. Courtoy, Y .A. DeClerck, E. Marbaix (2006). Mechanisms of pericyte recruitment in tumour angiogenesis: A new role for metalloproteinases. European J. Cancer, 42 (2006), 310-318. 
[11] M.A.J. Chaplain, G. Lolas. Mathematical modelling of cancer cell invasion of tissue: The role of the urokinase plasminogen activator system. Math. Mod. Meth. Appl. Sci., 11 (2005), 1685-1734.

[12] M. Ciofalo, M.W. Collins, T.R. Hennessy. "Microhydrodynamics phenomena in the circulation.” In: Nanoscale fluid dynamics in physiological processes: A review study. WIT Press, Southampton,1999, pp 219-236.

[13] G.E. Davis, K.A. Pintar Allen, R. Salazar, S.A. Maxwell. Matrix metalloproteinase-1 and -9 activation by plasmin regulates a novel endothelial cell-mediated mechanism of collagen gel contraction and capillary tube regression in three-dimensional collagen matrices. J. Cell Sci., 114 (2000), 917-930.

[14] A.W. El-Kareh, T.W. Secomb Theoretical models for drug delivery to solid tumours. Crit. Rev. Biomed. Eng., 25 (1997), 503-571.

[15] J. Folkman, M. Klagsbrun. Angiogenic factors. Science, 235 (1987), 442-447.

[16] Y.C. Fung. Biomechanics. Springer-Verlag, New-York, 1993.

[17] M.S. Gee, W.N. Procopio, S. Makonnen, M.D. Feldman, N.W. Yeilding, W.M.F. Lee. Tumor vessel development and maturation impose limits on the effectiveness of anti-vascular therapy. Am. J. Path., 162 (2003), 183-193.

[18] R. Gödde, H. Kurz. Structural and biophysical simulation of angiogenesis and vascular remodeling. Developmental Dynamics, 220 (2001), 387-401.

[19] M. Hidalgo, S.G. Eckkhardt. Development of matrix metalloproteinase inhibitors in cancer therapy. Journal of the National Cancer Institute, 93 (2001), 178-193.

[20] S. Hughes, T. Gardiner, P. Hu, L. Baxter, E. Rosinova, T. Chan-Ling. Altered pericyteendothelial relations in the rat retina during aging: Implications for vessel stability. Neurobiology of Aging, 27 (2006), 1838-1847.

[21] Y. Izumi. Tumour biology: herceptin acts as an antiangiogenic cocktail. Nature 416 (2002), 279-280.

[22] T.L. Jackson, S.R. Lubkin, J.D. Murray. Theoretical analysis of conjugate localization in two-step cancer chemotherapy. J. Math. Biol. 39 (1999), 353-376.

[23] R.K. Jain. (2003). Molecular regulation of vessel maturation. Nat. Med., 9 (2003), 685-93.

[24] A. Kamiya, R. Bukhari, T. Togawa. Adaptive regulation of wall shear stress optimizing vascular tree function. Bull. Math. Biology. 46 (1984), 127-137.

[25] G.S. Krenz, C.A. Dawson. Vessel distensibility and flow distribution in vascular trees. J. Math. Biol., 44 (2002), 360-374. 
[26] C.C. Kumar. Targeting integrins $\alpha v \beta 3$ and $\alpha v \beta 5$ for blocking tumour-induced angiogenesis. Adv. Exp. Med. Biol., 476 (2000), 169-180.

[27] H.A. Levine, S. Pamuk, B.D. Sleeman, M. Nielsen-Hamilton. Mathematical modeling of the capillary formation and development in tumor angiogenesis: penetration into the stroma. Bull. Math. Biol., 63 (2001), 801-863.

[28] S.R. McDougall, A.R.A. Anderson, M.A.J. Chaplain, J.A. Sherratt. Mathematical modelling of flow through vascular networks: implications for tumour-induced angiogenesis and chemotherapy strategies. Bull. Math. Biol., 64 (2002), 673-702.

[29] S.R. McDougall, A.R.A. Anderson, M.A.J. Chaplain. Mathematical modelling of dynamic adaptive tumour-induced angiogenesis: clinical implications and therapeutic targeting strategies. J. Theor. Biol., 241 (2006), 564-589.

[30] J.A. Madri, B.M. Pratt. Endothelial cell-matrix interactions: in vitro models of angiogenesis. J. Histochem. Cytochem. 34 (1986), 85-91.

[31] M.R. Mancuso et al. Rapid vascular regrowth in tumors after reversal of VEGF inhibition. J. Clin. Investigation, 116 (2006), 2610-2621.

[32] S. Morikawa, P. Baluk, T. Kaidoh, A. Haskell, R.K. Jain, D.M. McDonald. Abnormalities in pericytes on blood vessels and endothelial sprouts in tumors. Am. J. Path., 160 (2002), 985-1000.

[33] L.L. Munn. Aberrant vascular architecture in tumors and its importance in drug-based therapies. Drug Discovery Today, 8 (2003), 396-403.

[34] N. Paweletz, M. Knierim. Tumor-related angiogenesis. Crit. Rev. Oncol. Hematol. 9 (1989), 197-242.

[35] A.R. Pries, T.W. Secomb, P. Gaehtgens. Biophysical aspects of blood flow in the microvasculature. Cardiovasc. Res., 32 (1996), 654-667.

[36] A.R. Pries, T.W. Secomb, P. Gaehtgens. Structural adaptation and stability of microvascular networks: theory and simulation. Am. J. Physiol., 275 (1998), H349-H360.

[37] A.R. Pries, B. Reglin, T.W. Secomb. Structural adaptation of microvascular networks: functional roles of adaptive responses. Am. J. Physiol., 281 (2001a), H1015-H1025.

[38] A.R. Pries, B. Reglin, T.W. Secomb. Structural adaptation of vascular networks: role of the pressure response. Hypertension, 38 (2001b), 1476-1479.

[39] A. Quarteroni, M. Tuveri, A. Veneziani. Computational vascular fluid dynamics: problems, models and methods. Comput. Visual. Sci., 2 (2000), 163-197. 
[40] S. Rafil. Vascular and haematopoietic stem cells: novel targets for anti-angiogenesis therapy? Nature Reviews Cancer, 2 (2002), 826-835.

[41] C. Rouget. Memoire sur le developpement, la structure et les proprietes physiologiques des capillaires sanguins et lymphatiques. Arch. Physiol. Norm. Pathol., 5 (1873), 603-663.

[42] T.W. Secomb. Mechanics of blood flow in the microcirculation. In "Biological Fluid Dynamics.” eds. C.P. Ellington and T.J. Pedley. Company of Biologists, Cambridge, 1995, pp. 305-321.

[43] G.I. Schoefl. Studies of inflammation III. Growing capillaries: Their structure and permeability. Virchows Arch. Path. Anat., 337 (1963), 97-141.

[44] M.M. Sholley, G.P. Ferguson, H.R. Seibel, J.L. Montour, J.D. Wilson. Mechanisms of neovascularization. Vascular sprouting can occur without proliferation of endothelial cells. Lab. Invest., 51 (1984), 624-634.

[45] A. Stéphanou, S.R. McDougall, A.R.A. Anderson, M.A.J. Chaplain. Mathematical modelling of flow in $2 D$ and $3 D$ vascular networks: applications to anti-angiogenic and chemotherapeutic drug strategies. Math. Comp. Model., 41 (2005a), 1137-1156.

[46] A. Stéphanou, S.R. McDougall, A.R.A. Anderson, M.A.J. Chaplain. Mathematical modelling of the influence of blood rheological properties upon adaptive tumour-induced angiogenesis. Math. Comp. Model., 44 (2005b), 96-123.

[47] M.D. Sternlicht, Z. Werb. How matrix metalloproteinases regulate cell behavior. Annu. Rev. Cell Dev. Biol., 17 (2001), 463-516.

[48] G.M. Tozer, C. Kanthou, B.C. Baguley. Disrupting tumour blood vessels. Nature Reviews Cancer, 5 (2005), 423-433.

[49] L. Yan, M.A. Moses, S. Huang, D. Ingber (2000) Adhesion-dependent control of matrix metalloproteinase-2 activation in human capillary endothelial cells. J. Cell Sci., 113 (2000), 3979-3987. 